\title{
Pacific
}

Journal of

Mathematics

SINGULAR HOMOLOGY AND COHOMOLOGY WITH LOCAL COEFFICIENTS AND DUALITY FOR MANIFOLDS

EDWIN SPANIER 


\title{
SINGULAR HOMOLOGY AND COHOMOLOGY WITH LOCAL COEFFICIENTS AND DUALITY FOR MANIFOLDS
}

\section{E. SPANIER}

\begin{abstract}
This article contains an application of the author's previous work on cohomology theories on a space to an exposition of singular theory. After a summary of the relevant concepts concerning cohomology theories in general, singular homology and singular cohomology with local coefficients are defined. Each of these is presented in two versions, one with compact supports and one with arbitrary closed supports. It is shown that each version satisfies an appropriate duality theorem for arbitrary (i.e. nonorientable) topological manifolds.
\end{abstract}

1. Introduction. This paper is a presentation of singular homology and cohomology theory with local coefficients. Included is a treatment of the usual singular homology with compact supports (which is based on finite chains) and the singular homology based on locally finite chains. The former is a weakly additive theory and the latter is an additive theory.

Similarly, there are two types of singular cohomology, one with compact supports and one with arbitrary supports. In an $n$-manifold $X$ the basic duality theorem asserts the isomorphism of the two types of $q$-dimensional homology for an open pair $(U, V)$ in $X$ to the corresponding two types of $(n-q)$-dimensional cohomology of the complementary closed pair $(X-V, X-U)$ with coefficient systems suitably related.

Our approach is to study homology and cohomology on a fixed space $X$ and to prove the duality theorem referred to above by comparing two cohomology theories on $X$, one being the appropriate homology of the open pair in complementary dimension and the other being the corresponding cohomology theory of the complementary closed pair. For this we present the relevant concepts concerning such theories and a review of the comparison theorem for them.

Thus, the paper is divided into two parts, $\S \S 2$ through 5 are devoted to general concepts concerning covariant and contravariant functors defined on pairs in a space, and $\S \S 6$ through 10 are devoted to applications of these ideas to singular homology and cohomology and to a proof of the duality theorem for manifolds. 
Many of the results in the paper seem to be known in some form but not readily available in the literature. What is new is the generality of our treatment and the methods used. The presentation is reasonably self contained except for two results. The first is the main comparison theorem (stated below in Theorem 2.1) whose proof can be found in various forms in any of the references $[9,12,13]$. The second is the existence of a Thom class for arbitrary manifolds (needed in $\S 10$ for the duality theorems) a proof of which can be found in [7].

Section 2 contains definitions of a cohomology (homology) functor on $X$ as a contravariant (covariant) functor from closed (open) pairs in $X$ to the category of graded modules, together with a suitable natural transformation, such that continuity, excision, and exactness are satisfied. The main comparison theorem for cohomology functors is stated as well as a dual for homology functors.

In $\S 3$ we review some notation and terminology for chain (cochain) complexes, and in $\S 4$ we consider chain (cochain) functors on a space. These are often used in constructions to obtain homology (cohomology) functors. In $\S 5$ we introduce chain (cochain) prefunctors. These may be obtained by applying the hom functor to cochain (chain) functors. By taking suitable direct limits of a prefunctor one obtains a corresponding functor. Since the hom functor converts direct sums of modules to direct products, it takes weakly additive functors into additive prefunctors.

In $\S 6$ we define the singular chain complex with a local system as coefficients. This is a weakly additive theory. In $\S 7$ we consider locally finite singular chains and obtain an additive singular chain functor with local coefficients. We prove that in a locally compact finite polyhedron the corresponding homology is isomorphic to the cellular homology of the polyhedron based on infinite chains. In $\S 8$ we introduce singular cohomology with local coefficients. By using the hom functor on singular cochains with compact support we construct another additive homology functor. This is compared with the one based on locally finite singular chains, and the two are shown to be isomorphic on manifolds.

Sections 9 and 10 are devoted to a proof of the duality theorem for manifolds. The algebraic machinery necessary to compare singular homology of an open pair in $X$ with cohomology of the complementary closed pair is set up in $\S 9$. This uses a suitable cohomology class $U$ in $(X \times X, X \times X-\delta(X))$ where $\delta(X)$ is the diagonal of $X \times X$. In $\S 10$ we consider the case where $X$ is an $n$ manifold and $U$ is its 
Thom class and deduce the duality theorem. Some variants of duality are also discussed.

2. Cohomology and homology functors. We assume throughout that the space $X$ is paracompact and Hausdorff. This isn't absolutely essential for some of the results but it simplifies the presentation and suffices for our applications. $R$ will denote a fixed principal ideal domain. All modules will be over $R$.

A cohomology functor $H^{*}, \delta^{*}$ on $X$ consists of:

(a) a contravariant functor $H^{*}$ from the category of closed pairs $(A, B)$ in $X$ and inclusion maps between them to the category of graded $R$ modules $\left(H^{*}(A, B)=\left\{H^{q}(A, B)\right\}_{q \in \mathbf{Z})}\right.$ and homomorphisms of degree 0 between them, and

(b) for every closed triple $(A, B, C)$ in $X$ a natural transformation

$$
\delta^{*}: H^{*}(B, C) \rightarrow H^{*}(A, B) \text { of degree } 1,
$$

such that the following three properties are valid:

Continuity. For every closed pair $(A, B)$ in $X$ there is an isomorphism

$\rho: \lim _{\longrightarrow}\left\{H^{*}(M, N) \mid(M, N)\right.$ a closed neighborhood of $\left.(A, B)\right\}$

$$
\approx H^{*}(A, B)
$$

where $\rho\{u\}=u \mid(A, B)$ for $u \in H^{*}(M, N)$.

Excision. For closed sets $A, B$ in $X$ there is an isomorphism

$$
\rho: H^{*}(A \cup B, B) \approx H^{*}(A, A \cap B)
$$

where $\rho(u)=u \mid(A, A \cap B)$ for $u \in H^{*}(A \cup B, B)$.

Exactness. For every closed triple $(A, B, C)$ in $X$ the following sequence is exact

$$
\cdots \rightarrow H^{q}(A, B) \stackrel{\rho}{\rightarrow} H^{q}(A, C) \stackrel{\rho^{\prime}}{\rightarrow} H^{q}(B, C) \stackrel{\delta^{*}}{\rightarrow} H^{q+1}(A, B) \rightarrow \cdots
$$

What is here called continuity was called tautness in $[8,9]$. This definition of a cohomology functor is equivalent to that of an ES Theory $[10,11,12]$ although it is formally different. The definition given here is more convenient for dualization. It is a consequence that every cohomology functor defines a cohomology theory 
$H^{\prime}, \delta^{\prime}$ on $X$ (as defined in $\left.[9,12]\right)$ in which $H^{\prime}(A)=H^{*}(A, \varnothing)$ and $\delta^{\prime}: H^{\prime}(A \cap B) \rightarrow H^{\prime}(A \cup B)$ is suitably defined. The cohomology functor is nonnegative if $H^{q}(A, B)=0$ for $q<0$ and all closed pairs $(A, B)$.

A family $\left\{S_{j}\right\}_{j \in J}$ of subsets of $X$ is discrete if each point of $X$ has a neighborhood meeting at most one element of the family. Given a discrete family $\left\{\left(A_{j}, B_{j}\right)\right\}_{j \in J}$ of closed pairs in $X$, for each $j \in J$ let $C_{j}=\bigcup_{i \neq j} A_{i}$, a closed set in $X$. For each $j \in J$ there are restriction homomorphisms

$$
H^{*}\left(A_{j} \cup C_{j}, B_{j} \cup C_{j}\right) \stackrel{\rho_{j}^{\prime}}{\rightarrow} H^{*}\left(\bigcup_{j \in J}\left(A_{j}, B_{j}\right)\right) \stackrel{\rho_{\rho}}{\rightarrow} H^{*}\left(A_{j}, B_{j}\right) .
$$

The cohomology functor is said to be weakly additive if the homomorphisms $\left\{\rho_{j}^{\prime}\right\}$ define an isomorphism

$$
l: \bigoplus_{j \in J} H^{*}\left(A_{j} \cup C_{j}, B_{j} \cup C_{j}\right) \approx H^{*}\left(\bigcup_{j \in J}\left(A_{j}, B_{j}\right)\right)
$$

for every discrete family $\left\{\left(A_{j}, B_{j}\right)\right\}_{j \in J}$, and it is additive if the homomorphisms $\left\{\rho_{j}\right\}$ define an isomorphism

$$
\sigma: H^{*}\left(\bigcup_{j \in J}\left(A_{j}, B_{j}\right)\right) \approx \prod_{j \in J} H^{*}\left(A_{j}, B_{j}\right)
$$

for every discrete family $\left\{\left(A_{j}, B_{j}\right)\right\}_{j \in J}$. (Note that, by excision, $H^{*}\left(A_{j} \cup C_{j}, B_{j} \cup C_{j}\right)=H^{*}\left(A_{j} \cup\left(B_{j} \cup C_{j}\right), B_{j} \cup C_{j}\right) \approx H^{*}\left(A_{j}, B_{j}\right)$ for each $j \in J$, so weak additivity as defined above is equivalent to that defined in [13].)

A homomorphism $\varphi: H_{1}^{*}, \delta_{1}^{*} \rightarrow H_{2}^{*}, \delta_{2}^{*}$ between two cohomology functors on the same space $X$ is a natural transformation from $H_{1}^{*}$ to $H_{2}^{*}$ (of degree 0 ) which commutes up to sign with $\delta_{1}^{*}, \delta_{2}^{*}$. The following is a consequence of the main comparison theorem for cohomology theories $[9,12,13]$ and the five lemma.

THeOREM 2.1. Let $\varphi: H_{1}^{*}, \delta_{1}^{*} \rightarrow H_{2}^{*}, \delta_{2}^{*}$ be a homomorphism-between cohomology functors on $X$ and suppose both are weakly additive or both are additive. Suppose there is $n$ such that $\varphi: H_{1}^{*}(x, \varnothing) \rightarrow$ $H_{2}^{*}(x, \varnothing)$ is an n-equivalence for all $x \in X$. If both $H_{1}^{*}, \delta_{1}^{*}$ and $H_{2}^{*}, \delta_{2}^{*}$ are nonnegative or if $X$ is locally finite dimensional, then 
$\varphi: H_{1}^{*}(A, B) \rightarrow H_{2}^{*}(A, B)$ is an n-equivalence for all closed $(A, B)$ in $X$.

Dually, a homology functor $H_{*}, \partial_{*}$ on $X$ consists of:

$\left(\mathbf{a}^{\prime}\right)$ a convariant functor $H_{*}$ from the category of open pairs $(U, V)$ in $X$ to the category of graded $R$-modules $\left(H_{*}(U, V)=\right.$ $\left.\left\{H_{q}(U, V)\right\}_{q \in \mathbb{Z}}\right)$, and

$\left(\mathrm{b}^{\prime}\right)$ for every open triple $(U, V, W)$ in $X$ a natural transformation $\partial_{*}: H_{*}(U, V) \rightarrow H_{*}(V, W)$ of degree -1 ,

such that the following three properties are valid:

Continuity. For every open pair $(U, V)$ there is an isomorphism

$$
\begin{gathered}
i: \underset{\lim }{\longrightarrow}\left\{H_{*}\left(U^{\prime}, V^{\prime}\right) \mid\left(U^{\prime}, V^{\prime}\right) \text { open and }\left(\bar{U}^{\prime}, \bar{V}^{\prime}\right) \subset(U, V)\right\} \\
\\
\approx H_{*}(U, V)
\end{gathered}
$$

where $i\{z\}=i^{\prime}(z)$ for $z \in H_{*}\left(U^{\prime}, V^{\prime}\right)$ (and $i^{\prime}: H_{*}\left(U^{\prime}, V^{\prime}\right) \rightarrow$ $H_{*}(U, V)$ is induced by the inclusion map $\left.\left(U^{\prime}, V^{\prime}\right) \subset(U, V)\right)$.

Excision. For open $U, V$ in $X$ there is an isomorphism

$$
i: H_{*}(U, U \cap V) \approx H_{*}(U \cup V, V)
$$

induced by the inclusion $(U, U \cap V) \subset(U \cup V, V)$.

Exactness. For every open triple $(U, V, W)$ the following sequence is exact

$$
\cdots \rightarrow H_{q}(V, W) \stackrel{i}{\rightarrow} H_{q}(U, W) \stackrel{i^{\prime}}{\rightarrow} H_{q}(U, V) \stackrel{\partial_{*}}{\rightarrow} H_{q-1}(V, W) \rightarrow \cdots .
$$

The homology functor is nonnegative if $H_{q}(U, V)=0$ for $q<0$ and all open pairs $(U, V)$. Given a discrete family $\left\{\left(U_{j}, V_{j}\right)\right\}_{j \in J}$ of open pairs in $X$, for $j \in J$ let $W_{j}=\bigcup_{i \neq j} U_{i}$. For each $j \in J$ there are homomorphisms induced by inclusion

$$
H_{*}\left(U_{j}, V_{j}\right) \stackrel{i_{j}}{\rightarrow} H_{*}\left(\bigcup_{j \in J}\left(U_{j}, V_{j}\right)\right) \stackrel{i_{j}^{\prime}}{\rightarrow} H_{*}\left(U_{j} \cup W_{j}, V_{j} \cup W_{j}\right) .
$$

The homology functor is weakly additive if the homomorphisms $\left\{i_{j}\right\}$ define an isomorphism

$$
i: \bigoplus_{j \in J} H_{*}\left(U_{j}, V_{j}\right) \approx H_{*}\left(\bigcup_{j \in J}\left(U_{j}, V_{j}\right)\right)
$$


for every discrete family $\left\{\left(U_{j}, V_{j}\right)\right\}_{j \in J}$, and it is additive if the homomorphisms $\left\{i_{j}^{\prime}\right\}$ define an isomorphism

$$
\sigma^{\prime}: H_{*}\left(\bigcup_{j \in J}\left(U_{j}, V_{j}\right)\right) \approx \prod_{j \in J} H_{*}\left(U_{j} \cup W_{j}, V_{j} \cup W_{j}\right)
$$

for every discrete family $\left\{\left(U_{j}, V_{j}\right)\right\}_{j \in J}$.

Complementation and sign changing interchanges homology functors and cohomology functors. That is, the equation

$$
H_{q}(X-B, X-A)=H^{-q}(A, B)
$$

can be used to define a covariant functor $H_{*}$ on open pairs if $H^{*}$ is given on closed pairs, or conversely, defines $H^{*}$ on closed pairs if $H_{*}$ is given on open pairs. In each case there is a similar way to relate $\delta^{*}$ and $\partial_{*}$ so that corresponding to a homology functor $H_{*}, \partial_{*}$ there is a cohomology functor and conversely. Complementation in this form does not preserve nonnegativity but does preserve weak additivity and additivity and it is involutive.

A homomorphism $h: H_{*}, \partial_{*} \rightarrow H_{*}^{\prime}, \partial_{*}^{\prime}$ between two homology functors on the same space is a natural transformation from $H_{*}$ to $H_{*}^{\prime}$ commuting up to sign with $\partial_{*}$ and $\partial_{*}^{\prime}$. The following comparison theorem is one consequence of Theorem 2.1 obtained by complementation and sign changing.

THEOREM 2.2. Let $h: H_{*}, \partial_{*} \rightarrow H_{*}^{\prime}, \partial_{*}^{\prime}$ be a homomorphism between two homology functors on $X$ and suppose that both are weakly additive or both are additive. If $h: H_{*}(X, X-x) \rightarrow H_{*}^{\prime}(X, X-x)$ is an isomorphism for all $x \in X$ and $X$ is locally finite dimensional, then $h: H_{*}(U, V) \rightarrow H_{*}^{\prime}(U, V)$ is an isomorphism for every open pair $(U, V)$ in $X$.

3. Chain complexes. In the next section we will see that a homology (or cohomology) functor can be obtained from a functor from open (or closed) pairs to the category of chain (or cochain) complexes having properties analogous to the continuity, excision, and exactness properties.

In this section we summarize some definitions and properties of chain complexes over $R$. By changing the sign of the degree in such a complex we obtain a cochain complex and vice versa. This procedure will be referred to as "the sign changing trick" and implies that results valid for chain (or cochain) complexes have analogues valid for cochain (or chain) complexes. 
A chain transformation between two chain complexes is called a weak chain equivalence [3] if it induces isomorphisms of the respective homology modules. Every chain equivalence is a weak chain equivalence, and every weak chain equivalence between free chain complexes is a chain equivalence (recall that $R$ was assumed to be a principal ideal domain). In a similar fashion we define the concept of weak cochain equivalence between cochain complexes.

Let $\theta: C \rightarrow C^{\prime}$ be a chain transformation and let $G$ be an $R$ module. Then $\theta \otimes 1: C \otimes G \rightarrow C^{\prime} \otimes G$ is a chain transformation, and if $\theta$ is a chain equivalence, so is $\theta \otimes 1$. However, if $\theta$ is a weak chain equivalence, then $\theta \otimes 1$ need not be a weak chain equivalence. We will replace $C \otimes G$ by another chain complex which is a functor of $C$ and $G$ such that a weak chain equivalence of $C$ will induce a weak chain equivalence on the new chain complex. The main interest in this construction is in the case of chain complexes which are not free.

Let $0 \rightarrow P_{1} \stackrel{\partial}{\rightarrow} P_{0} \stackrel{\varepsilon}{\rightarrow} G \rightarrow 0$ be a free presentation of $G$. Then $P=\left\{P_{0}, P_{1}, \partial\right\}$ is a free chain complex with

$$
H_{q}(P) \approx \begin{cases}0 & \text { if } q \neq 0, \\ G & \text { if } q=0,\end{cases}
$$

and there is a weak chain equivalence $\varepsilon: P \rightarrow(G, 0)$ where $(G, 0)$ is the chain complex with $G$ in degree 0 and trivial chain modules in degrees other than 0 . If $P^{\prime}$ is another free chain complex with $\varepsilon^{\prime}: P^{\prime} \rightarrow(G, 0)$ a weak chain equivalence, there is a chain equivalence $\tau: P \rightarrow P^{\prime}$ such that $\varepsilon^{\prime} \circ \tau=\varepsilon$.

Consider the complex $C \otimes P$. Since $P$ is free it is a consequence of the Künneth formula [8] that for every $q$ there is a split short exact sequence (universal coefficient formula)

$$
0 \rightarrow H_{q}(C) \otimes G \rightarrow H_{q}(C \otimes P) \rightarrow \operatorname{tor}\left(H_{q-1}(C), G\right) \rightarrow 0 .
$$

Furthermore, if $\theta: C \rightarrow C^{\prime}$ is a weak chain equivalence, so is $\theta \otimes$ $1: C \otimes P \rightarrow C^{\prime} \otimes P$, and if $C$ is a free chain complex, then $1 \otimes \varepsilon: C \otimes$ $P \rightarrow C \otimes G$ is a weak chain equivalence. Finally, if $P^{\prime}$ is another free chain complex with $\varepsilon^{\prime}: P^{\prime} \rightarrow(G, 0)$ a weak chain equivalence, then $C \otimes P$ and $C \otimes P^{\prime}$ are chain equivalent. Therefore, $H_{*}(C \otimes P)$ depends canonically on $C$ and $G$.

If $C, C^{\prime}$ are chain complexes there is a chain complex hom $\left(C, C^{\prime}\right)$ [3] where $\operatorname{hom}\left(C, C^{\prime}\right)_{q}$ is the module of homomorphisms $\varphi$ from $C$ to $C^{\prime}$ of degree $q$ (so $\varphi\left(C_{i}\right) \subset C_{i+q}^{\prime}$ for all $i$ ) and with

$$
\partial^{\prime \prime}: \operatorname{hom}\left(C, C^{\prime}\right)_{q} \rightarrow \operatorname{hom}\left(C, C^{\prime}\right)_{q-1}
$$


defined by $\partial^{\prime \prime}(\varphi)=\partial^{\prime} \circ \varphi+(-1)^{q} \varphi \circ \partial$. Then $\partial^{\prime \prime} \partial^{\prime \prime}=0$ so $\operatorname{hom}\left(C, C^{\prime}\right)$ is a chain complex. Note that $\operatorname{hom}(C,(G, 0))_{q}=\operatorname{hom}\left(C_{-q}, G\right)$ so that if $C$ is a nonnegative chain complex, $\operatorname{hom}(C,(G, 0))$ is a nonpositive chain complex. In this case we consider $\operatorname{hom}(C,(G, 0))$ as a nonnegative cochain complex by changing the sign of the degree. Similarly if $C^{*}$ is a nonnegative cochain complex we change the sign of its degree and obtain a nonnegative chain complex $\operatorname{hom}\left(C^{*},(G, 0)\right)$.

If $C, C^{\prime}$ are chain complexes and $\theta: C \rightarrow C^{\prime}$ is a weak chain equivalence, then

$$
\operatorname{hom}(\theta, 1): \operatorname{hom}\left(C^{\prime},(G, 0)\right) \rightarrow \operatorname{hom}(C,(G, 0))
$$

need not be a weak cochain equivalence. Because of this we consider an injective resolution of $G$

$$
0 \rightarrow G \stackrel{\eta}{\rightarrow} Q^{0} \stackrel{\delta}{\rightarrow} Q^{1} \rightarrow 0 .
$$

Here, $Q=\left\{Q^{0}, Q^{1}, \delta\right\}$ is an injective cochain complex with

$$
H^{q}(Q) \approx \begin{cases}0, & q \neq 0 \\ G, & q=0\end{cases}
$$

and $\eta:(G, 0) \rightarrow Q$ is a weak cochain equivalence.

Consider the cochain complex $\operatorname{hom}(C, Q)$ (hom $(C, Q)^{q}$ consists of pairs $\left(\varphi_{0}, \varphi_{1}\right)$ where $\varphi_{0}: C_{q} \rightarrow Q^{0}$ and $\varphi_{1}: C_{q-1} \rightarrow Q^{1}$ and $\left.\delta\left(\varphi_{0}, \varphi_{1}\right)=\left((-1)^{q} \varphi_{0} \circ \partial,(-1)^{q} \varphi_{1} \circ \partial+\delta \circ \varphi_{0}\right)\right)$. For every $q$ there is a split short exact sequence (universal coefficient formula)

$$
0 \rightarrow \operatorname{ext}\left(H_{q-1}(C), G\right) \rightarrow H^{q}(\operatorname{hom}(C, Q)) \rightarrow \operatorname{hom}\left(H_{q}(C), G\right) \rightarrow 0 .
$$

Furthermore, if $\theta: C \rightarrow C^{\prime}$ is a weak chain equivalence so is $\operatorname{hom}(\theta, 1): \operatorname{hom}\left(C^{\prime}, Q\right) \rightarrow \operatorname{hom}(C, Q)$, and if $C$ is a free cochain complex, then

$$
\operatorname{hom}(1, \eta): \operatorname{hom}(C,(G, 0)) \rightarrow \operatorname{hom}(C, Q)
$$

is a weak cochain equivalence. Finally if $Q^{\prime}$ is another injective cochain complex with a weak cochain equivalence $\eta^{\prime}:(G, 0) \rightarrow Q^{\prime}$, then there is a cochain equivalence $\operatorname{hom}(C, Q) \rightarrow \operatorname{hom}\left(C, Q^{\prime}\right)$. Therefore, $H^{*}(\operatorname{hom}(C, Q))$ depends canonically on $C$ and $G$. Similarly if $C^{*}$ is a cochain complex then $\operatorname{hom}\left(C^{*}, Q\right)$ is a chain complex (with hom $\left(C^{*}, Q\right)_{q}$ consisting of pairs $\left(\psi_{0}, \psi_{1}\right)$ where $\psi_{0}: C^{q} \rightarrow Q^{0}$ and $\left.\psi_{1}: C^{q+1} \rightarrow Q^{1}\right)$. 
4. Chain and cochain functors. It is frequently the case that a homology (cohomology) functor is obtained from a suitable chain (cochain) functor on the space. This section contains the relevant definitions.

A chain functor $C_{*}$ on a topological space $X$ is a covariant functor from the category of open pairs in $X$ to the category of chain complexes of $R$ modules $\left(C_{*}(U, V)=\left\{C_{q}(U, V)\right\}_{q \in \mathbb{Z}}\right)$ such that the following three properties are valid:

Continuity. For every open pair $(U, V)$ there is a weak chain equivalence

$$
\begin{gathered}
i: \underset{\lim }{\longrightarrow}\left\{C_{*}\left(U^{\prime}, V^{\prime}\right) \mid\left(U^{\prime}, V^{\prime}\right) \text { open and }\left(\bar{U}^{\prime}, \bar{V}^{\prime}\right) \subset(U, V)\right\} \\
\rightarrow C_{*}(U, V) .
\end{gathered}
$$

Excision. For open sets $U, V$ in $X$ there is a weak chain equivalence

$$
i: C_{*}(U, U \cap V) \rightarrow C_{*}(U \cup V, V) .
$$

Exactness. For every open triple $(U, V, W)$ in $X$ there is a short exact sequence

$$
0 \rightarrow C_{*}(V, W) \stackrel{i}{\rightarrow} C_{*}(U, W) \stackrel{i^{\prime}}{\rightarrow} C_{*}(U, V) \rightarrow 0 .
$$

The chain functor is nonnegative if $C_{q}(U, V)=0$ for $q<0$ and all open pairs $(U, V)$ in $X$. It is weakly additive if for every discrete family $\left\{\left(U_{j}, V_{j}\right)\right\}_{j \in J}$ of open pairs in $X$ there is a weak chain equivalence

$$
i: \bigoplus_{j \in J} C_{*}\left(U_{j}, V_{j}\right) \rightarrow C_{*}\left(\bigcup_{j \in J}\left(U_{j}, V_{j}\right)\right)
$$

induced by the maps $i_{j}: C_{*}\left(U_{j}, V_{j}\right) \rightarrow C_{*}\left(\bigcup_{j \in J}\left(U_{j}, V_{j}\right)\right)$. It is additive if for every discrete family $\left\{\left(U_{j}, V_{j}\right)\right\}_{j \in J}$ there is a weak chain equivalence

$$
\sigma^{\prime}: C_{*}\left(\bigcup_{j \in J}\left(U_{j}, V_{j}\right)\right) \rightarrow \prod_{j \in J} C_{*}\left(U_{j} \cup W_{j}, V_{j} \cup W_{j}\right)
$$

where $W_{j}=\bigcup_{i \neq j} U_{i}$ and $\sigma^{\prime}$ is induced by the maps

$$
i_{j}^{\prime}: C_{*}\left(\bigcup_{j \in J}\left(U_{j}, V_{j}\right)\right) \rightarrow C_{*}\left(U_{j} \cup W_{j}, U_{j} \cup V_{j}\right)
$$


THEOREM 4.1. If $C_{*}$ is a chain functor on $X$ and $G$ is an $R$ module, there is a homology functor $H_{*}(\cdot, \cdot ; G)$ on $X$ with $H_{q}(U, V ; G)$ $=H_{q}\left(C_{*}(U, V) \otimes P\right)$ (where $P$ is a free resolution of $G$ ) and $\partial_{*}$ the connecting homomorphism corresponding to the exact sequence

$$
0 \rightarrow C_{*}(V, W) \otimes P \rightarrow C_{*}(U, W) \otimes P \rightarrow C_{*}(U, V) \otimes P \rightarrow 0 .
$$

If $C_{*}$ is nonnegative or weakly additive, the same is true of $H_{*}(\cdot, \cdot ; G)$, $\partial_{*}$.

Proof. The operation on chain complexes of forming their tensor product with $P$ commutes with direct sums and direct limits, takes weak chain equivalences into weak chain equivalences, and takes short exact sequences into short exact sequences. Therefore, the continuity, excision, and exactness properties of $C_{*}$ yield the corresponding properties of $H_{*}(\cdot, \cdot ; G), \partial_{*}$. Nonnegativity of $C_{*}$ clearly implies nonnegativity of $H_{*}(\cdot, \cdot ; G)$ and weak additivity of $C_{*}$ implies weak additivity of $H_{*}(\cdot, \cdot ; G)$.

Additivity of $C_{*}$ does not imply additivity of $H_{*}(\cdot, \cdot ; G)$ because tensor product of chain complexes with $P$ does not commute with infinite products.

Thus, weakly additive homology functors can be obtained from weakly additive chain functors. To get additive homology functors, in the next section we shall use the $\operatorname{hom}(\cdot, Q)$ construction applied to a weakly additive cochain functor. Therefore, we now introduce the concept of a cochain functor, dual to that of a chain functor.

A cochain functor $C^{*}$ on $X$ is a contravariant functor from closed pairs in $X$ to the category of cochain complexes of $R$ modules $\left(C^{*}(A, B)=\left\{C^{q}(A, B)\right\}_{q \in \mathbb{Z}}\right)$ such that the following properties are valid:

Continuity. For every closed pair $(A, B)$ there is a weak cochain equivalence

$\rho: \lim _{\longrightarrow}\left\{C^{*}(M, N) \mid(M, N)\right.$ a closed neighborhood of $\left.(A, B)\right\}$ $\rightarrow C^{*}(A, B)$.

Excision. For closed sets $A, B$ in $X$ there is a weak cochain equivalence

$$
\rho: C^{*}(A \cup B, B) \rightarrow C^{*}(A, A \cup B) .
$$


Exactness. For every closed triple $(A, B, C)$ in $X$ there is a short exact sequence

$$
0 \rightarrow C^{*}(A, B) \stackrel{\rho}{\rightarrow} C^{*}(A, C) \stackrel{\rho^{\prime}}{\rightarrow} C^{*}(B, C) \rightarrow 0 .
$$

The cochain functor is nonnegative if $C^{q}(A, B)=0$ for $q<0$ and all closed $(A, B)$ in $X$. It is weakly additive if for every discrete family $\left\{\left(A_{j}, B_{j}\right)\right\}_{j \in J}$ of closed pairs in $X$ the homomorphisms

$$
\rho_{j}^{\prime}: C^{*}\left(A_{j} \cup C_{j}, B_{j} \cup C_{j}\right) \rightarrow C^{*}\left(\bigcup_{j \in J}\left(A_{j}, B_{j}\right)\right)
$$

induce a weak cochain equivalence

$$
\imath: \bigoplus_{j \in J} C^{*}\left(A_{j} \cup C_{j}, B_{j} \cup C_{j}\right) \rightarrow C_{*}\left(\bigcup_{j \in J}\left(A_{j}, B_{j}\right)\right)
$$

(where $C_{j}=\bigcup_{i \neq j} A_{i}$ for each $j$ ), and it is additive if there is a weak cochain equivalence

$$
\sigma: C^{*}\left(\bigcup_{j \in J}\left(A_{j}, B_{j}\right)\right) \rightarrow \prod_{j \in J} C^{*}\left(A_{j}, B_{j}\right)
$$

induced by the maps $\rho_{j}: C^{*}\left(\bigcup_{j \in J}\left(A_{j}, B_{j}\right)\right) \rightarrow C^{*}\left(A_{j}, B_{j}\right)$.

Complementations and sign changing

$$
\left(C^{q}(A, B)=C_{-q}(X-B, X-A)\right)
$$

interchanges chain and cochain functors preserving weak additivity and additivity (but not nonnegativity). The following analogue of Theorem 4.1 is obtained by complementation and sign changing.

THEOREM 4.2. If $C^{*}$ is a cochain functor on $X$ and $G$ is an $R$ module, there is a cohomology functor $H^{*}(\cdot, \cdot ; G), \delta^{*}$ on $X$ with $H^{q}(A, B ; G)=H^{q}\left(C^{*}(A, B) \otimes P\right)$ (where $P$ is a free resolution of $G)$ and $\delta^{*}$ is the connecting homomorphism corresponding to the exact sequence

$$
0 \rightarrow C^{*}(A, B) \otimes P \rightarrow C^{*}(A, C) \otimes P \rightarrow C^{*}(B, C) \otimes P \rightarrow 0 .
$$

If $C^{*}$ is weakly additive the same is true of $H^{*}(\cdot, \cdot ; G)$.

5. Prefunctors. We have viewed homology on $X$ as a covariant functor on open pairs of $X$ and so our chain functors are also covariant functors on open pairs of $X$. Dually cohomology on $X$ is a 
contravariant functor from closed pairs of $X$ and the corresponding cochain functors are also contravariant functors on closed pairs. It may happen that we encounter or construct a covariant functor from closed pairs of $X$ or a contravariant functor from open pairs. If such functors satisfy suitable hypotheses they are called chain (cochain) prefunctors on $X$. By passing to appropriate direct limits they give rise to chain (cochain) functors.

One way of constructing a chain (cochain) prefunctor is to apply $\operatorname{hom}(\cdot, Q)$ to a cochain (chain) functor (where $Q$ is an injective resolution of a module $G$ ). This procedure applied to a weakly additive cochain (chain) functor yields an additive chain (cochain) prefunctor.

A cochain prefunctor $C^{*}$ on $X$ is a contravariant functor from the category of open pairs $(U, V)$ of $X$ to the category of cochain complexes of $R$ modules $\left(C^{*}(U, V)=\left\{C^{q}(U, V)\right\}_{q \in \mathbb{Z}}\right)$ such that the following are valid:

Excision. For open sets $U, V$ in $X$ there is a weak cochain equivalence

$$
\rho: C^{*}(U \cup V, V) \rightarrow C^{*}(U, U \cap V) .
$$

Exactness. For every open triple $(U, V, W)$ in $X$ there is a short exact sequence

$$
0 \rightarrow C^{*}(U, V) \stackrel{\rho}{\rightarrow} C^{*}(U, W) \stackrel{\rho^{\prime}}{\rightarrow} C^{*}(V, W) \rightarrow 0 .
$$

Nonnegativity, weak additivity, and additivity are defined for cochain prefunctors in fashion analogous to their definition for cochain functors. There is no continuity property involved in the definition of cochain prefunctor.

Dually a chain prefunctor $C_{*}$ on $X$ is a covariant functor $C_{*}$ from the category of closed pairs $(A, B)$ in $X$ to the category of chain complexes of $R$ modules satisfying excision and exactness.

THeOREM 5.1. Let $C_{*}$ be a chain functor on $X$ and $G$ an $R$ module. For an open $(U, V)$ in $X$ define

$$
C^{*}(U, V)=\operatorname{hom}\left(C_{*}(U, V), Q\right)
$$

where $Q$ is an injective resolution of $G$. Then $C^{*}$ is a cochain prefunctor on $X$. If $C_{*}$ is weakly additive, then $C^{*}$ is additive. Dually; if $C^{*}$ is a (weakly additive) chain functor define, for a closed pair $(A, B)$ in $X$,

$$
C_{*}(A, B)=\operatorname{hom}\left(C^{*}(A, B), Q\right) .
$$

Then $C_{*}(A, B)$ is an (additive) chain prefunctor on $X$. 
Proof. Because $Q$ is an injective complex, the excision property of $C_{*}$ implies that of $C^{*}$ and the exactness property of $C_{*}$ implies that of $C^{*}$. Thus, $C^{*}$ is a cochain prefunctor.

Assume $C_{*}$ is weakly additive. We prove $C^{*}$ is additive. Let $\left\{\left(U_{j}, V_{j}\right)\right\}_{j \in J}$ be a discrete family of open pairs. Then there is a weak chain equivalence

$$
i: \bigoplus_{j \in J} C_{*}\left(U_{j}, V_{j}\right) \rightarrow C_{*}\left(\bigcup_{j \in J}\left(U_{j}, V_{j}\right)\right) \text {. }
$$

Therefore, there is a weak cochain equivalence

$$
\begin{gathered}
\operatorname{hom}(i, 1): \operatorname{hom}\left(C_{*}\left(\bigcup_{j \in J}\left(U_{j}, V_{j}\right)\right), Q\right) \\
\rightarrow \operatorname{hom}\left(\bigoplus_{j \in J} C_{*}\left(U_{j}, V_{j}\right), Q\right) .
\end{gathered}
$$

Since $\operatorname{hom}\left(\bigoplus_{j \in J} C_{*}\left(U_{j}, V_{j}\right), Q\right) \approx \prod_{j \in J} \operatorname{hom}\left(C_{*}\left(U_{j}, V_{j}\right), Q\right)$ we obtain a weak chain equivalence

$$
\sigma: C^{*}\left(\bigcup_{j \in J}\left(U_{j}, V_{j}\right)\right) \rightarrow \prod_{j \in J} C^{*}\left(U_{j}, V_{j}\right)
$$

proving that $C^{*}$ is additive.

This completes the proof of the statement about the cochain prefunctor. The dual statement about chain prefunctors is proved similarly.

Theorem 5.2. Let $C^{*}$ be a cochain prefunctor. Define, for $(A, B)$ a closed pair in $X$,

$\bar{C}^{*}(A, B)=\lim _{\longrightarrow}\left\{C^{*}(U, V) \mid(U, V)\right.$ an open neighborhood of $\left.(A, B)\right\}$.

Then $\bar{C}^{*}$ is a cochain functor and if $C^{*}$ is additive, so is $\bar{C}^{*}$. Similarly if $C_{*}$ is a chain prefunctor define, for open $(U, V)$,

$$
\underline{C}_{*}(U, V)=\lim _{\longrightarrow}\left\{C_{*}(A, B) \mid(A, B) \text { closed } \subset(U, V)\right\} .
$$

Then $\underline{C}_{*}$ is a chain functor and if $C_{*}$ is additive, so is $\underline{C}_{*}$.

Proof. Since $X$ is normal, if a closed pair $(A, B)$ is contained in an open pair $(U, V)$ there is a closed pair of neighborhoods $(M, N)$ 
of $(A, B)$ with $(M, N) \subset(U, V)$. Using this and the definition of $\bar{C}^{*}$ it follows that $\bar{C}^{*}$ is continuous.

Since the cohomology of a direct limit of cochain complexes is the direct limit of the cohomology of the cochain complexes, the excision property for $\bar{C}^{*}$ follows from that for $C^{*}$.

Since the direct limit of exact sequences is exact, the exactness property for $\bar{C}^{*}$ follows from that for $C^{*}$.

Therefore, $\bar{C}^{*}$ is a cochain functor. To prove it is additive if $C^{*}$ is, note that, since $X$ is paracompact, it is collectionwise normal. Hence, if $\left\{\left(A_{j}, B_{j}\right)\right\}_{j \in J}$ is a discrete family of closed pairs in $X$ there exist discrete families of open pairs $\left\{\left(U_{j}, V_{j}\right)\right\}_{j \in J}$ with $\left(A_{j}, B_{j}\right) \subset$ $\left(U_{j}, V_{j}\right)$ for each $j$, and as these discrete families vary over such neighborhoods their unions $\bigcup_{j \in J}\left(U_{j}, V_{j}\right)$ form a cofinal family of open neighborhoods of $\bigcup_{j \in J}\left(A_{j}, B_{j}\right)$. Since $C^{*}$ is additive, there is a weak cochain equivalence

$$
\sigma: C^{*}\left(\bigcup_{j \in J}\left(U_{j}, V_{j}\right)\right) \rightarrow \prod_{j \in J} C^{*}\left(U_{j}, V_{j}\right) .
$$

Taking the direct limit of both sides as $\left(U_{j}, V_{j}\right)$ vary over discrete neighborhoods of $\left(A_{j}, B_{j}\right)$ yields the additivity of $\bar{C}^{*}$. This proves the result for $\bar{C}^{*}$.

The result for $\underline{C}_{*}$ follows similarly.

Combining Theorems 5.1 and 5.2 we obtain the following.

Corollary 5.3. Let $C_{*}$ be a chain functor on $X$ and $G$ an $R$ module. For a closed pair $(A, B)$ in $X$ define

$$
\bar{C}^{*}(A, B ; G)=\underline{\lim }\left\{\operatorname{hom}\left(C_{*}(U, V), Q\right) \mid(U, V)\right. \text { an open }
$$
neighborhood of $(A, B)\}$

where $Q$ is an injective resolution of $G$. Then $\bar{C}^{*}(\cdot, \cdot ; G)$ is a cochain functor on $X$. If $C_{*}$ is weakly additive, then $\bar{C}^{*}(\cdot, \cdot ; G)$ is additive. Dually, if $C^{*}$ is a (weakly additive) cochain functor on $X$ define, for $(U, V)$ open in $X$,

$$
\underline{C}_{*}(U, V ; G)=\underline{\lim }\left\{\operatorname{hom}\left(C^{*}(A, B), Q\right) \mid(A, B) \text { closed } \subset(U, V)\right\} \text {. }
$$

Then $\underline{C}_{*}$ is an (additive) homology functor on $X$.

REMARK 5.4. In Corollary 5.3 if $C_{*}\left(\right.$ or $C^{*}$ ) is nonnegative it is not true that $\bar{C}^{*}$ (or $\underline{C}_{*}$ ) is nonnegative; however, the corresponding 
cohomology functor $\bar{H}^{*}$ (or homology functor $\underline{H}_{*}$ ) determined by $\bar{C}^{*}\left(\underline{C}_{*}\right)$ is nonnegative because of the universal coefficient formula.

Even for prefunctors which are neither weakly additive nor additive we can obtain weakly additive functors by using limits over compact (or cobounded) sets as we now describe. (Recall that a set $A \subset X$ is cobounded if $X-A$ has compact closure.)

THEOREM 5.5. Let $C^{*}$ be a cochain prefunctor on $X$. For $(A, B)$ a closed pair in $X$ define

$$
\bar{C}_{c}^{*}(A, B)=\lim _{\longrightarrow}\left\{C^{*}(U, V) \mid(U, V)\right. \text { open cobounded }
$$

neighborhood of $(A, B)\}$.

Then $\bar{C}_{c}^{*}$ is a weakly additive cochain functor. Dually, if $C_{*}$ is a chain prefunctor on $X$ define, for open $(U, V)$ in $X$,

$$
\underline{C}_{*}^{c}(U, V)=\lim _{\longrightarrow}\left\{C_{*}(A, B) \mid(A, B) \text { compact } \subset(U, V)\right\} .
$$

Then $\underline{C}_{*}^{c}$ is a weakly additive chain functor on $X$.

Proof. The proof that $\bar{C}_{c}^{*}$ is a cochain functor (and $\underline{C}_{*}^{c}$ is a chain functor) is analogous to the proof in Theorem 5.2 that $\bar{C}^{*}\left(\underline{C}_{*}\right)$ is a cochain (chain) functor.

We show $\bar{C}_{c}^{*}$ is weakly additive. Let $\left\{\left(A_{j}, B_{j}\right)\right\}_{j \in J}$ be a discrete family of closed pairs in $X$ and let $(U, V)$ be an open cobounded neighborhood of $\bigcup_{j \in J}\left(A_{j}, B_{j}\right)$. Then there is a finite set $F \subset J$ such that $j \notin F$ implies $A_{j} \subset V$ (since $X-V$ is compact it can meet only finitely many $A_{j}$ 's). If $u \in \bar{C}_{c}^{*}\left(\bigcup_{j \in J}\left(A_{j}, B_{j}\right)\right)$ has the form $u=\{v\}$ where $v \in C^{*}(U, V)$ then $u \mid \bigcup_{j \notin F}\left(A_{j}, B_{j}\right)=0$ so (by the analogue of the Lemma in [13]) it follows that $\bar{C}_{c}^{*}$ is weakly additive.

6. Singular homology. In this section we introduce the usual singular homology of $X$ with coefficients in a local system. This is a weakly additive homology functor on $X$. A corresponding additive homology functor will be introduced in the next section. Our treatment of singular theory dates back to Eilenberg [5] and is the one most commonly used since the appearance of [5].

We begin by recalling some properties of local systems. Local systems were defined by Steenrod [14]. The definitions below are equivalent to his. A local system [8] $\Gamma$ of $R$ modules on a space $X$ is a function associating to every $x \in X$ an $R$ module $\Gamma_{x}$ and to every path $\omega: I \rightarrow X$ a homomorphism $\Gamma_{\omega}: \Gamma_{\omega(0)} \rightarrow \Gamma_{\omega(1)}$ (this is the 
reverse of the definition in [8]) such that:

(1) If $\varepsilon_{x}$ is the constant path at $x$, then $\Gamma_{\varepsilon_{x}}=1_{\Gamma(x)}$.

(2) If $\omega(1)=\omega^{\prime}(0)$ the product path $\omega * \omega^{\prime}$ is defined and $\Gamma_{\omega * \omega^{\prime}}=$ $\Gamma_{\omega^{\prime}} \circ \Gamma_{\omega}: \Gamma_{\omega(0)} \rightarrow \Gamma_{\omega^{\prime}(1)} \cdot$

(3) If $\omega$ and $\omega^{\prime}$ are homotopic paths (i.e. homotopic relative to $\{0,1\})$, then $\Gamma_{\omega}=\Gamma_{\omega^{\prime}}: \Gamma_{\omega(0)} \rightarrow \Gamma_{\omega(1)}$.

Let $f: Y \rightarrow X$ be a continuous map and $\Gamma$ a local system on $X$. A $\Gamma$ section of $f$ is a function $s$ assigning to every $y \in Y$ an element $s(y) \in \Gamma_{f(y)}$ such that, for every path $\omega$ in $Y, \Gamma_{f \circ \omega}(s(\omega(0)))=$ $s(\omega(1))$. The set of all such $\Gamma$ sections of $f$ is an $R$ module $\Gamma(f)$ under pointwise operations of functions. In case $Y$ is path connected and every closed path in $Y$ is mapped by $f$ into a null homotopic path in $X$ (e.g. if $Y$ is simply connected), then for every $y_{0} \in Y$ the map $\varphi_{0}: \Gamma(f) \rightarrow \Gamma_{f\left(y_{0}\right)}$ defined by $\varphi_{0}(s)=s\left(y_{0}\right)$ is an isomorphism.

If $\Gamma$ is a local system and $G$ is an $R$ module, $\operatorname{hom}(\Gamma, G)$ is the local system $(\operatorname{hom}(\Gamma, G))_{x}=\operatorname{hom}\left(\Gamma_{x}, G\right)$ and, for $\omega$ a path in $X,(\operatorname{hom}(\Gamma, G))_{\omega}:(\operatorname{hom}(\Gamma, G))_{\omega(0)} \rightarrow(\operatorname{hom}(\Gamma, G))_{\omega(1)}$ is equal to $\left(\operatorname{hom}\left(\Gamma_{\omega}, 1\right)\right)^{-1}: \operatorname{hom}\left(\Gamma_{\omega(0)}, G\right) \rightarrow \operatorname{hom}\left(\Gamma_{\omega(1)}, G\right)$ (so for $\varphi \in$ $\left.\operatorname{hom}(\Gamma, G)_{\omega(0)},(\operatorname{hom}(\Gamma, G))_{\omega}(\varphi)=\varphi \circ \Gamma_{\omega}^{-1} \in \operatorname{hom}(\Gamma, G)_{\omega(1)}\right)$.

Two local systems $\Gamma$ and $\Gamma^{\prime}$ are paired to an $R$ module $G$ if there is bilinear map $\langle\cdot, \cdot\rangle: \Gamma_{x} \otimes \Gamma_{x}^{\prime} \rightarrow G$ for each $x$ such that if $\omega$ is a path in $X$ then for $\gamma \in \Gamma_{\omega(0)}, \gamma \in \Gamma_{\omega(0)}^{\prime}$ we have $\left\langle\gamma, \gamma^{\prime}\right\rangle=\left\langle\Gamma_{\omega}(\gamma), \Gamma_{\omega}^{\prime}\left(\gamma^{\prime}\right)\right\rangle$.

EXAMPLE 6.1. There is a pairing of $\operatorname{hom}(\Gamma, G)$ and $\Gamma$ to $G$ defined by $\langle\varphi, \gamma\rangle=\varphi(\gamma)$ for $\gamma \in \Gamma_{x}$ and $\varphi \in(\operatorname{hom}(\Gamma, G))_{x}=\operatorname{hom}\left(\Gamma_{x}, G\right)$.

If $\Gamma$ and $\Gamma$ are paired to $G$ then for every path connected $Y$ and map $f: Y \rightarrow X$ there is a pairing of $\Gamma(f)$ and $\Gamma^{\prime}(f)$ to $G$ defined by $\left\langle s, s^{\prime}\right\rangle=\left\langle s(y), s^{\prime}(y)\right\rangle$ for $y \in Y$ (the value of $\left\langle s(y), s^{\prime}(y)\right\rangle$ is independent of $y \in Y$ because $Y$ is path connected).

For $q<0$ define $\Delta_{q}(X ; \Gamma)=0$ and for $q \geq 0$ define $\Delta_{q}(X ; \Gamma)$ to be set of all finitely non-zero functions $c$ which assign to every singular $q$-simplex $\sigma: \Delta^{q} \rightarrow X$ an element $c(\sigma) \in \Gamma(\sigma)$. Then $\Delta_{q}(X ; \Gamma)$ is an $R$ module under pointwise operations of functions. If $g_{\sigma} \in \Gamma(\sigma)$ let $g_{\sigma} \sigma$ denote the element of $\Delta_{q}(X ; \Gamma)$ such that

$$
g_{\sigma}\left(\sigma^{\prime}\right)= \begin{cases}0 & \text { if } \sigma \neq \sigma^{\prime} \\ g_{\sigma} & \text { if } \sigma=\sigma^{\prime}\end{cases}
$$

Then every element $c \in \Delta_{q}(X ; \Gamma)$ has the form $c=\sum_{\sigma} g_{\sigma} \sigma$ where $g_{\sigma}=0$ except for a finite set of $\sigma$ 's.

If $g_{\sigma} \in \Gamma(\sigma)$ and $\sigma^{(i)}$ is the $i$ th face of $\sigma$, then $g_{\sigma} \mid \sigma^{(i)} \in \Gamma\left(\sigma^{(i)}\right)$. 
Thus, there is a homomorphism

$$
\partial: \Delta_{q}(X ; \Gamma) \rightarrow \Delta_{q-1}(X ; \Gamma) \text { for } q>0
$$

defined by $\partial\left(\sum_{\sigma} g_{\sigma} \sigma\right)=\sum_{\sigma} \sum_{0<i<q}(-1)^{i}\left(g_{\sigma} \mid \sigma^{(i)}\right) \sigma^{(i)}$. It is easily verified that $\partial \partial=0$ so that $\Delta_{*}(X ; \Gamma)=\left\{\Delta_{q}(X ; \Gamma), \partial\right\}$ is a nonnegative chain complex. If $c=\sum_{\sigma} g_{\sigma} \sigma$ its support $|c|=\bigcup\left\{\sigma\left(\Delta^{q}\right) \mid g_{\sigma} \neq 0\right\}$, a compact subset of $X$. If $A \subset X$, then $\Delta_{*}(A ; \Gamma)=\left\{c \in \Delta_{*}(X ; \Gamma) \| c \mid \subset\right.$ $A\}$ is a subcomplex of $\Delta_{*}(X ; \Gamma)$, and we define

$$
\Delta_{*}(X, A ; \Gamma)=\Delta_{*}(X ; \Gamma) / \Delta_{*}(A ; \Gamma)
$$

so there is a short exact sequence

$$
0 \rightarrow \Delta_{*}(A ; \Gamma) \rightarrow \Delta_{*}(X ; \Gamma) \rightarrow \Delta_{*}(X, A ; \Gamma) \rightarrow 0 .
$$

THeOREM 6.2. Let $\Gamma$ be a fixed local system on $X$. Then $\Delta_{*}(\cdot, \cdot)$ defined for $(U, V)$ open by $\Delta_{*}(U, V)=\Delta_{*}(U, V ; \Gamma)$ is a nonnegative weakly additive chain functor on $X$.

Proof. Continuity follows from the fact that as $\left(U^{\prime}, V^{\prime}\right)$ vary over open sets with $\left(\bar{U}^{\prime}, \bar{V}^{\prime}\right) \subset(U, V)$, then $U \Delta_{*}\left(U^{\prime} ; \Gamma\right)=\Delta_{*}(U ; \Gamma)$ and $U \Delta_{*}\left(V^{\prime} ; \Gamma\right)=\Delta_{*}(V ; \Gamma)$ because every element of $\Delta_{*}(U ; \Gamma)$ (or $\left.\Delta_{*}(V ; \Gamma)\right)$ has support a compact subset of $U$ (or $V$ ) so is contained in $U^{\prime}$ (or $V^{\prime}$ ) for some open $U^{\prime}$ (or $V^{\prime}$ ) whose closure is contained in $U$ (or $V$ ).

For excision let $\mathscr{V}$ be a collection of subsets of $X$ and define $\Delta_{*}(\mathscr{V} ; \Gamma)$ to be the subcomplex of $\Delta_{*}(X ; \Gamma)$ consisting of finite sums $\sum c_{j}$ such that for each $j, c_{j} \in \Delta_{*}(X ; \Gamma)$ and there is some $V_{j} \in$ $\mathscr{V}$ with $\left|c_{j}\right| \subset V_{j}$. If $\mathscr{V}$ is a collection of subsets of $A$ such that $A=\bigcup_{V \in \mathscr{V}}$ int $V$, then the inclusion map $\Delta_{*}(\mathscr{V} ; \Gamma) \subset \Delta_{*}(A ; \Gamma)$ is a chain equivalence (proof analogous to that of Theorem 14 on $\mathrm{p}$. 178 of [8]). In particular if $U$ and $V$ are open sets in $X$, then $\Delta_{*}(U ; \Gamma)+\Delta_{*}(V ; \Gamma) \subset \Delta_{*}(U \cup V ; \Gamma)$ is a chain equivalence. It follows that

$$
\begin{aligned}
& {\left[\Delta_{*}(U ; \Gamma)+\Delta_{*}(V ; \Gamma)\right] / \Delta_{*}(V ; \Gamma) \rightarrow \Delta_{*}(U \cup V ; \Gamma) / \Delta_{*}(V ; \Gamma)} \\
& \quad=\Delta_{*}(U \cup V, V ; \Gamma)
\end{aligned}
$$

is a chain equivalence. Excision follows from this and the Noether isomorphism

$$
\begin{aligned}
\Delta_{*}(U, U \cap V ; \Gamma) & =\Delta_{*}(U ; \Gamma) / \Delta_{*}(U \cap V ; \Gamma) \\
& =\Delta_{*}(U ; \Gamma) /\left[\Delta_{*}(U ; \Gamma) \cap \Delta_{*}(V ; \Gamma)\right] \\
& \approx\left[\Delta_{*}(U ; \Gamma)+\Delta_{*}(V ; \Gamma)\right] / \Delta_{*}(V ; \Gamma) .
\end{aligned}
$$


If $(U, V, W)$ is an open triple in $X$ there is a short exact sequence

$$
0 \rightarrow \Delta_{*}(V, W ; \Gamma) \rightarrow \Delta_{*}(U, W ; \Gamma) \rightarrow \Delta_{*}(U, V ; \Gamma) \rightarrow 0
$$

so exactness is satisfied.

Thus, $\Delta_{*}(\cdot, \cdot)$ is a nonnegative (by definition) chain functor. It is weakly additive because every chain has compact supports [13].

It follows from Theorem 6.2 that for every $R$ module $G$ the singular homology on $X$ with coefficients $\Gamma \otimes G$, denoted by $H_{*}(\cdot, \cdot ; \Gamma \otimes G)$ and defined by $H_{q}(U, V ; \Gamma \otimes G)=H_{q}\left(\Delta_{*}(U, V ; \Gamma) \otimes P\right)$ (where $P$ is a projective resolution of $G$ ) is a nonnegative weakly additive homology functor on $X$.

7. Locally finite singular homology. In this section we introduce the locally finite singular chains to obtain an additive homology functor. In a special situation we relate this new homology to limits of the usual singular homology. This is applied to show that for a locally finite simplicial complex the locally finite singular homology is isomorphic to the simplicial homology based on infinite chains. The locally finite singular homology was considered in Séminaire Cartan [2] where it was called "singular homology of the second kind".

For $q \geq 0$ a function $c$ which assigns to every singular $q$-simplex $\sigma: \Delta^{q} \rightarrow X$ an element $c(\sigma) \in \Gamma(\sigma)$ is called a locally finite $q$-chain if $\left\{\sigma\left(\Delta^{q}\right) \mid c(\sigma) \neq 0\right\}$ is a locally finite family in $X$. The locally finite $q$-chains form an $R$ module $\Delta_{q}^{\infty}(X ; \Gamma)$ under the usual operations on functions. For $q<0$ we define $\Delta_{q}^{\infty}(X ; \Gamma)=0$. If $c \in \Delta_{q}^{\infty}(X ; \Gamma)$ with $0 \leq q$ its support $|c|=\bigcup\left\{\sigma\left(\Delta^{q}\right) \mid c(\sigma) \neq 0\right\}$, a closed subset of $X$. If $c \in \Delta_{q}^{\infty}(X ; \Gamma)$ with $q<0$ we define $|c|=\varnothing$.

If $\left\{c_{j}\right\}_{j \in J}$ is a family of elements of $\Delta_{q}^{\infty}(X ; \Gamma)$ it is said to be locally finite if the family of supports $\left\{\left|c_{j}\right|\right\}_{j \in J}$ is locally finite. In this case the sum $\sum_{j \in J} c_{j}$ is defined as an element of $\Delta_{q}^{\infty}(X ; \Gamma)$ (because for every singular $q$-simplex $\sigma, \sigma\left(\Delta^{q}\right)$ is compact so meets only finitely many $\left|c_{j}\right|$ and so $c_{j}(\sigma)=0$ except for a finite set of $j$ 's). In particular, if $q>0$ and $c=\sum_{\sigma} g_{\sigma} \sigma \in \Delta_{q}^{\infty}(X ; \Gamma)$, then $\left\{\sum_{0 \leq i \leq q}(-1)^{i}\left(g_{\sigma} \mid \sigma^{(i)}\right) \sigma^{(i)}\right\}_{g_{\sigma} \neq 0}$ is a locally finite family so

$$
\sum_{\sigma} \sum_{0 \leq i \leq q}(-1)^{i}\left(g_{\sigma} \mid \sigma^{(i)}\right) \sigma^{(i)} \in \Delta_{q-1}^{\infty}(X ; \Gamma)
$$

and there is a homomorphism

$$
\partial: \Delta_{q}^{\infty}(X ; \Gamma) \rightarrow \Delta_{q-1}^{\infty}(X ; \Gamma)
$$


such that $\partial\left(\sum_{\sigma} g_{\sigma} \sigma\right)=\sum_{\sigma} \sum_{0 \leq i \leq \sigma}(-1)^{i}\left(g_{\sigma} \mid \sigma^{(i)}\right) \sigma^{(i)}$. Then $|\partial c| \subset|c|$ and $\partial \partial c=0$ so there is a nonnegative chain complex $\Delta_{*}^{\infty}(X ; \Gamma)=$ $\left\{\Delta_{q}^{\infty}(X ; \Gamma), \partial\right\}$ of locally finite singular chains with coefficients $\Gamma$. We define $H_{q}^{\infty}(X ; \Gamma)=H_{q}\left(\Delta_{*}^{\infty}(X ; \Gamma)\right)$.

Clearly $\Delta_{*}(X ; \Gamma) \subset \Delta_{*}^{\infty}(X ; \Gamma)$ (in fact, $\Delta_{*}(X ; \Gamma)$ is the subcomplex of $\Delta_{*}^{\infty}(X ; \Gamma)$ of chains having compact support) so there is a homomorphism

$$
H_{q}(X ; \Gamma) \rightarrow H_{q}^{\infty}(X ; \Gamma) .
$$

If $A \subset X$ we define ${ }^{X} \Delta_{*}^{\infty}(A ; \Gamma)=\left\{c \in \Delta_{*}^{\infty}(X ; \Gamma) \| c \mid \subset A\right\}$. This is a subcomplex of $\Delta_{*}^{\infty}(X ; \Gamma)$ and consists of chains in $A$ which are locally finite in $X$ (a stronger condition than being locally finite in $A)$. We define ${ }^{X} H_{*}^{\infty}(A ; \Gamma)=H_{*}\left({ }^{X} \Delta_{*}^{\infty}(A ; \Gamma)\right)$. In case $A$ is closed in $X,{ }^{X} \Delta_{*}^{\infty}(A ; \Gamma)=\Delta_{*}^{\infty}(A ; \Gamma)$ so that ${ }^{X} H_{*}^{\infty}(A ; \Gamma)=H_{*}^{\infty}(A ; \Gamma)$ in this case.

If $c \in{ }^{X} \Delta_{*}^{\infty}(A ; \Gamma)$ there is a set $F$ closed in $X$ with $F \subset A$ such that $c \in \Delta_{*}^{\infty}(F ; \Gamma)$ (for example, $F=|c|$ ). Therefore, if $\left\{A_{j}\right\}_{j \in J}$ is a family of subsets of $A$ directed upward by inclusion such that every subset of $A$ which is closed in $X$ is contained in $A_{j}$ for some $j \in J$ then ${ }^{X} \Delta_{*}^{\infty}(A ; \Gamma)=\bigcup_{j \in J}{ }^{X} \Delta_{*}^{\infty}\left(A_{j} ; \Gamma\right)$ and so $\underline{\lim }\left\{{ }^{X} H_{*}^{\infty}\left(A_{j} ; \Gamma\right)\right\} \approx$ ${ }^{X} H_{*}^{\infty}(A ; \Gamma)$.

If $B \subset A \subset X$ then ${ }^{X} \Delta_{*}^{\infty}(B ; \Gamma) \subset{ }^{X} \Delta_{*}^{\infty}(A ; \Gamma)$ and we define ${ }^{X} \Delta_{*}^{\infty}(A, B ; \Gamma)={ }^{X} \Delta_{*}^{\infty}(A ; \Gamma) /{ }^{X} \Delta_{*}^{\infty}(B ; \Gamma)$. There is then a short exact sequence of chain complexes

$$
0 \rightarrow{ }^{x} \Delta_{*}^{\infty}(B ; \Gamma) \rightarrow{ }^{x} \Delta_{*}^{\infty}(A ; \Gamma) \rightarrow{ }^{x} \Delta_{*}^{\infty}(A, B ; \Gamma) \rightarrow 0 .
$$

THEOREM 7.1. For $(U, V)$ an open pair in $X$ define $\Delta_{*}^{\infty}(U, V)=$ ${ }^{X} \Delta_{*}^{\infty}(U, V ; \Gamma)$. Then $\Delta_{*}^{\infty}$ is a nonnegative additive chain functor on $X$.

Proof. Continuity follows from the fact that as $\left(U^{\prime}, V^{\prime}\right)$ vary over open pairs with $\left(\bar{U}^{\prime}, \bar{V}^{\prime}\right) \subset(U, V)$ then $U \Delta_{*}^{\infty}\left(U^{\prime}\right)=\Delta_{*}^{\infty}(U)$ and $\bigcup \Delta_{*}^{\infty}\left(V^{\prime}\right)=\Delta_{*}^{\infty}(V)$ so that $\lim _{\longrightarrow}\left\{\Delta_{*}^{\infty}\left(U^{\prime}, V^{\prime}\right)\right\} \approx \Delta_{*}^{\infty}(U, V)$.

For excision let $\mathscr{V}$ be a family of subsets of $X$ and define ${ }^{x} \Delta_{*}^{\infty}(\mathscr{V} ; \Gamma)$ to be the subcomplex of $\Delta_{*}^{\infty}(X ; \Gamma)$ consisting of locally finite sums $\sum_{j \in J} c_{j}$ such that for each $j \in J, c_{j} \in \Delta_{*}^{\infty}(X ; \Gamma)$ and there is some $V_{j} \in \mathscr{V}$ with $\left|c_{j}\right| \subset V_{j}$. If $\mathscr{V}$ is a collection of subsets of $A$ such that $A=\bigcup_{V \in \mathscr{V}}$ int $V$ then the inclusion map ${ }^{X} \Delta_{*}^{\infty}(\mathscr{V} ; \Gamma) \subset{ }^{X} \Delta_{*}^{\infty}(A ; \Gamma)$ is a chain equivalence (proof analogous to that of Theorem 14 on p. 178 of [8]). Excision follows from this (as in the proof of excision in Theorem 6.1). 
If $(U, V, W)$ is an open triple in $X$ there is a short exact sequence

$$
0 \rightarrow \Delta_{*}^{\infty}(V, W) \rightarrow \Delta_{*}^{\infty}(U, W) \rightarrow \Delta_{*}^{\infty}(U, V) \rightarrow 0
$$

so exactness is satisfied.

Thus, $\Delta_{*}^{\infty}$ is a nonnegative chain functor on $X$. To show it is additive suppose $\left\{U_{j}\right\}_{j \in J}$ is a discrete family of open sets in $X$ and $V \subset \bigcup_{j \in J} U_{j}$ is open. Then there are isomorphisms

$$
\begin{aligned}
{ }^{X} \Delta_{*}^{\infty}\left(\bigcup_{j \in J} U_{j} ; \Gamma\right) & \approx \prod_{j \in J}{ }^{X} \Delta_{*}^{\infty}\left(U_{j} ; \Gamma\right), \\
{ }^{X} \Delta_{*}^{\infty}(V ; \Gamma) & \approx \prod_{j \in J}{ }^{X} \Delta_{*}^{\infty}\left(V \cap U_{j} ; \Gamma\right)
\end{aligned}
$$

and additivity of $\Delta_{*}^{\infty}$ follows.

Thus, usual singular homology is obtained from a weakly additive chain functor and locally finite singular homology is obtained from an additive chain functor. In case $X$ is compact the two theories agree. The following is a generalization of this.

Lemma 7.2. If $B \subset A$ and $A-B$ has compact closure in $X$ then $\Delta_{*}(A, B ; \Gamma) \approx{ }^{X} \Delta_{*}^{\infty}(A, B ; \Gamma)$.

Proof. If $A-B$ has compact closure in $X$, then for $c \in$ ${ }^{X} \Delta_{q}^{\infty}(A, B ; \Gamma)$ the set $\left\{\sigma \mid c(\sigma) \neq 0\right.$ and $\left.\sigma\left(\Delta^{q}\right) \cap(A-B) \neq \varnothing\right\}$ is finite. Therefore, ${ }^{X} \Delta_{q}^{\infty}(A ; \Gamma)={ }^{X} \Delta_{q}^{\infty}(B ; \Gamma)+\Delta_{q}(A ; \Gamma)$ and so

$$
\begin{aligned}
{ }^{X} \Delta_{q}^{\infty}(A, B ; \Gamma) & ={ }^{X} \Delta_{q}^{\infty}(A ; \Gamma) /{ }^{X} \Delta_{q}^{\infty}(B ; \Gamma) \\
& =\left[{ }^{X} \Delta_{q}^{\infty}(B ; \Gamma)+\Delta_{q}(A ; \Gamma)\right] /{ }^{X} \Delta_{q}^{\infty}(B ; \Gamma) \\
& \approx \Delta_{q}(A ; \Gamma) /\left[{ }^{X} \Delta_{q}^{\infty}(B ; \Gamma) \cap \Delta_{q}(A ; \Gamma)\right] \\
& =\Delta_{q}(A ; \Gamma) / \Delta_{q}(B ; \Gamma)=\Delta_{q}(A, B ; \Gamma) .
\end{aligned}
$$

Our next result relates $H_{*}^{\infty}$ to limits of $H_{*}$ in a special situation.

TheOREM 7.3. Assume there is a sequence $\left\{C_{i}\right\}_{i \geq 0}$ of subsets of $X$ such that $X=\bigcup_{i}$ int $C_{i}, C_{i} \subset C_{i+1}$ for each $i$, and $\bar{C}_{i}$ is compact for each $i$. Then there is a short exact sequence

$$
\begin{aligned}
0 & \rightarrow \lim ^{1}\left\{H_{q+1}\left(X, X-C_{i} ; \Gamma\right)\right\} \\
& \rightarrow H_{q}^{\infty}(X ; \Gamma) \rightarrow \underset{\lim }{\longleftarrow}\left\{H_{q}\left(X, X-C_{i} ; \Gamma\right)\right\} \rightarrow 0 .
\end{aligned}
$$


Proof. (Note that a sequence $\left\{C_{i}\right\}$ of subsets of $X$ satisfying the hypotheses of the theorem exists if and only if $X$ is a $\sigma$-compact space as defined in Dugundji [4].) For each $i$ there is a quotient chain map

$$
\tau_{i}: \Delta_{*}^{\infty}(X ; \Gamma) \rightarrow{ }^{X} \Delta_{*}^{\infty}\left(X, X-C_{i} ; \Gamma\right)
$$

and these define a chain map

$$
\tau: \Delta_{*}^{\infty}(X ; \Gamma) \rightarrow \lim \left\{{ }^{X} \Delta_{*}^{\infty}\left(X, X-C_{l} ; \Gamma\right)\right\} .
$$

We prove $\tau$ is an isomorphism. If $c \in \Delta_{*}^{\infty}(X ; \Gamma)$ is in $\operatorname{ker} \tau$ then $\tau_{i}(c)=0$ for each $i$ so $c \in{ }^{X} \Delta_{*}^{\infty}\left(X-C_{i} ; \Gamma\right)$ for each $i$. Since $\bigcup_{i} C_{i}=X$, it follows that $\bigcap_{i}{ }^{X} \Delta_{*}^{\infty}\left(X-C_{i} ; \Gamma\right)=0$ so $c=0$ and $\tau$ is a monomorphism.

To show $\tau$ is an epimorphism let $\left\{c_{i} \in{ }^{X} \Delta_{*}^{\infty}\left(X, X-C_{i} ; \Gamma\right)\right\}$ be an element in $\lim _{\longleftarrow}\left\{{ }^{X} \Delta_{*}^{\infty}\left(X, X-C_{i} ; \Gamma\right)\right\}$. The element $c_{i}$ can be regarded as a locally finite sum of singular simplexes each having support which meets $C_{i}$. Similarly $c_{i+1}$ is a locally finite sum of singular simplexes each having support which meets $C_{i+1}$. The condition that $c_{i+1}$ maps to $c_{i}$ implies that on singular simplexes whose support meets $C_{i}$ both $c_{i}$ and $c_{i+1}$ have the same value. Therefore, there is a chain $C=\sum g_{\sigma} \sigma$ in $X$ such that if $|\sigma| \cap C_{i} \neq \varnothing$ then $g_{\sigma}=$ the value of $c_{i}$ on $\sigma$. We show $c$ is a locally finite chain in $X$. Since $X=\bigcup \operatorname{int} C_{i}$, if $x \in X$ there is $i$ such that $x \in \operatorname{int} C_{i}$. If $|\sigma| \cap$ int $C_{i} \neq \varnothing$ then $|\sigma| \cap C_{i} \neq \varnothing$ so $g_{\sigma}$ is the value of $c_{i}$ on $\sigma$. Since $c_{i}$ is a locally finite chain, there is a neighborhood $N$ of $x$ such that there are only a finite number of $\sigma$ 's with $|\sigma| \cap N \neq \varnothing$ and $c_{i}(\sigma) \neq 0$. Then $N \cap \operatorname{int} C_{i}$ is a neighborhood of $x$ such that there are only finitely many $\sigma$ 's with $|\sigma| \cap\left(N \cap \operatorname{int} C_{i}\right) \neq \varnothing$ and $g_{\sigma} \neq 0$. Therefore, $c \in \Delta_{*}^{\infty}(X ; \Gamma)$ and clearly $\tau(c)=\left\{\tau_{i}(c)\right\}=\left\{c_{i}\right\}$ so $\tau$ is an epimorphism.

By Lemma 7.2 since $\bar{C}_{i}$ is compact, there is an isomorphism $\Delta_{*}\left(X, X-C_{i} ; \Gamma\right) \approx{ }^{X} \Delta_{*}^{\infty}\left(X, X-C_{i} ; \Gamma\right)$ so that $\Delta_{*}^{\infty}(X ; \Gamma)$ is isomorphic to $\lim \left\{\Delta_{*}\left(X, X-C_{i} ; \Gamma\right)\right\}$. Each of the chain maps $\Delta_{*}\left(X, X-C_{i+1} ; \Gamma\right) \rightarrow \Delta_{*}\left(X, X-C_{i} ; \Gamma\right)$ is an epimorphism so by A.15 on p. 402 of [6], $\lim ^{1}\left\{\Delta_{*}\left(X, X-C_{i} ; \Gamma\right)\right\}=0$, and then by A.19 on p. 407 of [6] there is a short exact sequence

$$
\begin{aligned}
0 & \rightarrow \lim ^{1}\left\{H_{q+1}\left(X, X-C_{i} ; \Gamma\right)\right\} \\
& \rightarrow H_{q}^{\infty}(X ; \Gamma) \rightarrow \underset{\lim }{\longleftarrow}\left\{H_{q}\left(X, X-C_{i} ; \Gamma\right)\right\} \rightarrow 0 .
\end{aligned}
$$

We use this last result to show that for the space of a locally finite simplicial complex $K$, then $H_{q}^{\infty}(|K| ; \Gamma)$ is isomorphic to the 
homology group of the complex of infinite simplicial chains with coefficients $\Gamma$.

Let $K$ be a locally finite simplicial complex and let $C_{*}^{\infty}(K ; \Gamma)$ be the infinite chain complex of oriented simplexes with coefficients $\Gamma$ (thus an element $c \in C_{q}^{\infty}(K ; \Gamma)$ is a function assigning to every oriented $q$-simplex $\sigma$ of $K$ an element $c(\sigma) \in \Gamma(|\sigma|)$ such that if $\sigma^{\prime}$ is the oppositely oriented simplex then $\left.c(\sigma)+c\left(\sigma^{\prime}\right)=0\right)$. Similarly let $\Delta_{*}^{\infty}(K ; \Gamma)$ be the infinite chain complex of ordered simplexes with coefficients $\Gamma$ (so $c \in \Delta_{q}^{\infty}(K ; \Gamma$ ) is a function assigning to every ordered $q$-simplex $\sigma$ of $K$ an element $c(\sigma) \in \Gamma(|\sigma|))$. There are natural chain maps [8] $\mu^{\infty}: \Delta_{*}^{\infty}(K ; \Gamma) \rightarrow C_{*}^{\infty}(K ; \Gamma)$ and $\nu^{\infty}: \Delta_{*}^{\infty}(K ; \Gamma) \rightarrow \Delta_{*}^{\infty}(|K| ; \Gamma)$ which are chain equivalences for every finite complex $K$.

THEOREM 7.4. If $K$ is a locally finite simplicial complex there are isomorphisms

$$
\mu_{*}^{\infty}: H_{q}\left(\Delta_{*}^{\infty}(K ; \Gamma)\right) \approx H_{q}\left(C_{*}^{\infty}(K ; \Gamma)\right)
$$

and

$$
\nu_{*}^{\infty}: H_{q}\left(\Delta_{*}^{\infty}(K ; \Gamma)\right) \approx H_{q}^{\infty}(|K| ; \Gamma) .
$$

Proof. The local finiteness of $K$ implies that $K=\bigcup_{i=0}^{\infty} K_{i}$ where $K_{i}$ is finite for each $i$ and $\left|K_{i}\right| \subset$ int $\left|K_{i+1}\right|$. By analogues of Theorem 7.3 for $C_{*}^{\infty}(K ; \Gamma)$ and $\Delta_{*}^{\infty}(K ; \Gamma)$ there is a commutative diagram with exact rows (in which $K$-int $\left|K_{i}\right|$ is denoted by $L_{i}$ and all coefficients are in $\Gamma$ )

$$
\begin{aligned}
& 0 \rightarrow \lim ^{1}\left\{H_{q+1}\left(C_{*}\left(K, L_{i}\right)\right)\right\} \rightarrow H_{q}\left(C_{*}^{\infty}(K)\right) \rightarrow \underline{\lim }\left\{H_{q}\left(C_{*}\left(K, L_{l}\right)\right)\right\} \rightarrow 0 \\
& 0 \rightarrow \lim ^{1}\left\{H_{q+1}^{\mu_{*} \uparrow}\left(\Delta_{*}\left(K, L_{i}\right)\right)\right\} \rightarrow H_{q}\left(\Delta_{*}^{\infty} \uparrow(K)\right) \rightarrow \underset{\mu_{*} \uparrow}{\longleftarrow}\left\{H_{q}\left(\Delta_{*}\left(K, L_{l}\right)\right)\right\} \rightarrow 0 \\
& \left.0 \rightarrow \lim ^{1}\left\{H_{q+1}^{\nu_{*}} \downarrow|K|,\left|L_{l}\right|\right)\right\} \rightarrow H_{q}^{\nu_{*}^{\infty}}(|K|) \rightarrow \lim \left\{H_{q}\left(|K|,\left|L_{l}\right|\right)\right\} \rightarrow 0 .
\end{aligned}
$$

Since the vertical maps on the sides are known to be isomorphisms, the result follows from the 5-lemma.

8. Singular cohomology. In this section we define the singular cohomology of $X$ which coefficients in a local system, and, using this, we define another additive homology function $X$ which we compare with the one defined in the last section.

Let $\Gamma$ be a local system on $X$ and for a pair $(M, N)$ in $X$ let $\Delta^{q}(M, N ; \Gamma)=0$ if $q<0$ and for $q \geq 0$ let $\Delta^{q}(M, N ; \Gamma)$ be the module of $q$-cochains of $M$ which vanish on $N$ (i.e. $u \in$ $\Delta^{q}(M, N ; \Gamma)$ is a function assigning to every singular $q$-simplex 
$\sigma: \Delta^{q} \rightarrow M$ an element $u(\sigma) \in \Gamma(\sigma)$ such that if $\sigma\left(\Delta^{q}\right) \subset N$ then $u(\sigma)=0)$. For $u \in \Delta^{q}(M, N ; \Gamma), q \geq 0$ define $\delta u \in \Delta^{q+1}(M, N ; \Gamma)$ by

$$
(\delta u)(\sigma)=\sum_{0 \leq i \leq q+1}(-1)^{i} \overline{u\left(\sigma^{(i)}\right)}
$$

where $\sigma: \Delta^{q+1} \rightarrow M$ and if $g \in \Gamma\left(\sigma^{(i)}\right)$ then $\bar{g} \in \Gamma(\sigma)$ is the unique element such that $\bar{g} \mid \sigma^{(i)}=g$. Then $\delta \delta=0$ so there is a cochain complex $\Delta^{*}(M, N ; \Gamma)=\left\{\Delta^{q}(M, N ; \Gamma), \delta\right\}$.

In case $\Gamma, \Gamma^{\prime}$ are local systems on $X$ paired to an $R$ module $G$ as in $\S 6$ there is a pairing $\Delta^{q}(M, N ; \Gamma) \otimes \Delta_{q}\left(M, N ; \Gamma^{\prime}\right) \rightarrow G$ defined by $\langle u, c\rangle=\sum\left\langle u(\sigma), g_{\sigma}\right\rangle$ where $c=\sum_{\sigma} g_{\sigma} \sigma$. In case $u \in \Delta^{q}(M, N ; \Gamma)$ and $c=\sum g_{\sigma} \sigma \in \Delta_{q+1}\left(M, N ; \Gamma^{\prime}\right)$ we have

$$
\left.\langle\delta u, c\rangle=\sum_{g_{\sigma}}\left\langle\delta u(\sigma), g_{\sigma}\right\rangle=\sum_{\sigma} \sum_{0 \leq i \leq q+1}(-1)^{i} \overline{\left\langle u\left(\sigma^{i}\right)\right.}, g_{\sigma}\right\rangle .
$$

Now for any $g \in \Gamma\left(\sigma^{(i)}\right)$ we have $\left\langle\bar{g}, g_{\sigma}\right\rangle=\left\langle g, g_{\sigma} \mid \sigma^{(i)}\right\rangle$. Therefore,

$$
\begin{aligned}
\langle\delta u, c\rangle & =\sum_{\sigma} \sum_{0 \leq i \leq q+1}(-1)^{i}\left\langle u\left(\sigma^{(i)}\right), g_{\sigma} \mid \sigma^{(i)}\right\rangle \\
& =\sum_{\sigma}\left\langle u, \partial\left(g_{\sigma} \sigma\right)\right\rangle=\langle u, \partial c\rangle .
\end{aligned}
$$

In case $\Gamma=\operatorname{hom}\left(\Gamma^{\prime}, G\right)$ we have

$$
\Delta^{q}\left(M, N ; \operatorname{hom}\left(\Gamma^{\prime}, G\right)\right) \approx \operatorname{hom}\left(\Delta_{q}\left(M, N ; \Gamma^{\prime}\right), G\right)
$$

an $\delta$ corresponds to $\operatorname{hom}(\partial, 1)$ under the above pairing.

The singular cohomology $H^{q}(M, N ; \Gamma)$ is defined to equal $H^{q}\left(\Delta^{*}(M, N ; \Gamma)\right)$.

Consider the cochain complex $\Delta^{*}(U, V ; \Gamma)$ as a functor of open pairs $(U, V)$ in $X$. For an open triple $(U, V, W)$ there is a short exact sequence

$$
0 \rightarrow \Delta^{*}(U, V ; \Gamma) \rightarrow \Delta^{*}(U, W ; \Gamma) \rightarrow \Delta^{*}(V, W ; \Gamma) \rightarrow 0 .
$$

If $U, V$ are open sets in $X$ then (by an argument similar to the proof of excision in Theorem 6.2) there is a weak cochain equivalence

$$
\Delta^{*}(U \cup V, V ; \Gamma) \rightarrow \Delta^{*}(U, U \cap V ; \Gamma) .
$$

Furthermore, if $\left\{\left(U_{j}, V_{j}\right)\right\}_{j \in J}$ is a discrete family of open pairs in $X$ there is an isomorphism

$$
\Delta^{*}\left(\bigcup_{j \in J}\left(U_{j}, V_{j}\right) ; \Gamma\right) \approx \prod_{j \in J} \Delta^{*}\left(U_{j}, V_{j} ; \Gamma\right)
$$


Thus, $\Delta^{*}(\cdot, \cdot ; \Gamma)$ is a nonnegative additive cochain prefunctor on $X$.

By Theorem 5.2 there is a corresponding cochain functor $\bar{\Delta}^{*}$ which associates to a closed pair $(A, B)$ in $X$ the cochain complex

$$
\bar{\Delta}^{*}(A, G ; \Gamma)=\underset{\lim }{\longrightarrow}\left\{\Delta^{*}(U, V ; \Gamma) \mid(U, V)\right. \text { an open }
$$

neighborhood of $(A, B)\}$

whose cohomology functor is denoted by $\bar{H}^{*}(A, B ; \Gamma)$. It is a nonnegative additive cohomology functor on $X$. Note that $\bar{H}^{*}(A, B ; \Gamma)$ is not the singular cohomology of $(A, B)$ with coefficients $\Gamma$ but is the limit of the singular cohomology of open neighborhoods of $(A, B)$. (However; because $(X, \varnothing)$ is an open pair, $\bar{H}^{*}(X, \varnothing ; \Gamma)=$ $H^{*}(X, \varnothing ; \Gamma)$ is the singular cohomology of $(X, \varnothing)$.) In case $X$ is an HLC space, $\bar{H}^{*}(A, B ; \Gamma)$ is isomorphic to the Cech-Alexander cohomology of $(A, B)$ with coefficients $\Gamma$ [9].

Similarly by Theorem 5.5 there is a weakly additive cochain functor $\bar{\Delta}_{c}^{*}$ which assigns to a closed pair $(A, B)$ in $X$ the cochain complex

$$
\bar{\Delta}_{c}^{*}(A, B ; \Gamma)=\lim _{\longrightarrow}\left\{\Delta^{*}(U, V ; \Gamma) \mid(U, V)\right. \text { open cobounded }
$$

$$
\text { neighborhood of }(A, B)\}
$$

whose cohomology functor is denoted by $\bar{H}_{c}^{*}(A, B ; \Gamma)$. It is a nonnegative weakly additive cohomology functor on $X$. In case $X$ is HLC, $\bar{H}_{c}^{*}(A, B ; \Gamma)$ is isomorphic to the Čech-Alexander cohomology of $(A, B)$ with coefficients $\Gamma$ and with compact supports.

For a pair $(M, N)$ in $X$ we define

$$
\begin{aligned}
& \underline{\Delta}_{*}(M, N ; \operatorname{hom}(\Gamma, G)) \\
& \quad=\underset{\lim }{\longrightarrow}\left\{\operatorname{hom}\left(\bar{\Delta}_{c}^{*}(A, B ; \Gamma), Q\right) \mid(A, B) \text { closed } \subset(M, N)\right\}
\end{aligned}
$$

where $Q$ is, as usual, an injective resolution of $G$. We define $\underline{H}_{q}(M, N ; \operatorname{hom}(\Gamma, G))=H_{q}\left(\underline{\Delta}_{*}(M, N ; \operatorname{hom}(\Gamma, G))\right)$. Then for a closed pair $(A, B)$, we have

$$
\underline{\Delta}_{*}(A, B ; \operatorname{hom}(\Gamma, G))=\operatorname{hom}\left(\bar{\Delta}_{c}^{*}(A, B ; \Gamma), Q\right) .
$$

By Corollary 5.3 there is an additive chain functor $\underline{\Delta}_{*}$ which associates to an open pair $(U, V)$ in $X$ the chain complex $\underline{\Delta}_{*}(U, V ; \operatorname{hom}(\Gamma, G))$. We want to compare the additive homology functor

$$
\underline{H}_{*}(\cdot, \cdot ; \operatorname{hom}(\Gamma, G))
$$

just defined with the additive homology functor defined in $\S 7$ by using locally finite singular chains. Note that $\underline{\Delta}_{*}$ (and $\underline{H}_{*}$ ) are defined for 
local coefficients of the form $\operatorname{hom}(\Gamma, G)$ for some local system $\Gamma$ and some module $G$. It is not clear how much of a restriction this imposes on the resulting local system $\operatorname{hom}(\Gamma, G)$.

We begin with a pairing

$$
\Delta^{q}\left(U^{\prime}, V^{\prime} ; \Gamma\right) \times{ }^{X} \Delta_{q}^{\infty}(A, B ; \operatorname{hom}(\Gamma, G)) \rightarrow G
$$

for a closed pair $(A, B)$ contained in an open cobounded pair $\left(U^{\prime}, V^{\prime}\right)$. Let $c=\sum_{\sigma} g_{\sigma} \sigma \in{ }^{X} \Delta_{q}^{\infty}(A, B ; \operatorname{hom}(\Gamma, G))$ and let $u \in$ $\Delta^{q}\left(U^{\prime}, V^{\prime} ; \Gamma\right)$. Then $u$ vanishes on every singular simplex $\sigma$ in $V^{\prime}$. Since $X-V^{\prime}$ is compact there are only a finite number of $\sigma$ 's such that $g_{\sigma} \neq 0$ and $|\sigma| \cap\left(X-V^{\prime}\right) \neq \varnothing$. Therefore

$$
\langle u, c\rangle=\sum_{\sigma} g_{\sigma}(u(\sigma))
$$

is a finite sum of elements of $G$. In case

$$
c=\sum_{\sigma} g_{\sigma} \sigma \in{ }^{X} \Delta_{q+1}^{\infty}(A, B ; \operatorname{hom}(\Gamma, G))
$$

then $\partial c=\sum_{\sigma} \sum_{0 \leq i \leq q+1}(-1)^{i}\left(g_{\sigma} \mid \sigma^{(i)}\right) \sigma^{(i)}$ and

$$
\begin{aligned}
\langle u, \partial c\rangle & =\sum_{\sigma} \sum_{0 \leq i \leq q+1}(-1)^{i}\left(g_{\sigma} \mid \sigma^{(i)}\right)\left(u\left(\sigma^{(i)}\right)\right) \\
& =\sum_{\sigma} \sum_{0 \leq i \leq q+1}(-1)^{i} g_{\sigma}\left(\overline{u\left(\sigma^{(i)}\right)}\right) \\
& =\sum_{\sigma} g_{\sigma}(\delta u(\sigma))=\langle\delta u, c\rangle .
\end{aligned}
$$

Passing to the direct limit as $\left(U^{\prime}, V^{\prime}\right)$ varies over open cobounded neighborhoods of $(A, B)$ we obtain a pairing

$$
\bar{\Delta}_{c}^{q}(A, B ; \Gamma) \times{ }^{X} \Delta_{q}^{\infty}(A, B ; \operatorname{hom}(\Gamma, G)) \rightarrow G
$$

which corresponds to a homomorphism

$$
\varphi:{ }^{X} \Delta_{q}^{\infty}(A, B ; \operatorname{hom}(\Gamma, G)) \rightarrow \operatorname{hom}\left(\bar{\Delta}_{c}^{q}(A, B ; \Gamma), G\right)
$$

such that $(\varphi(c))(\{u\})=\langle u, c\rangle$ for $c \in{ }^{X} \Delta_{q}^{\infty}(A, B ; \operatorname{hom}(\Gamma, G))$ and $u \in \Delta^{q}\left(U^{\prime}, V^{\prime} ; \Gamma\right)$ where $\left(U^{\prime}, V^{\prime}\right)$ is an open cobounded neighborhood of $(A, B)$. For the injective resolution

$$
0 \rightarrow G \stackrel{\eta}{\rightarrow} Q^{0} \rightarrow Q^{1} \rightarrow 0
$$

we see that $\eta \varphi:{ }^{X} \Delta_{q}^{\infty}(A, B ; \operatorname{hom}(\Gamma, G)) \rightarrow \operatorname{hom}\left(\bar{\Delta}_{c}^{q}(A, B ; \Gamma), Q\right)$ and

$$
\begin{aligned}
(\eta \varphi)(\partial c)(\{u\}) & =\eta\langle u, \partial c\rangle=\eta\langle\delta u, c\rangle=(\eta \varphi)(c)(\{\delta u\}) \\
& =\partial(\eta \varphi(c))(\{u\})
\end{aligned}
$$

so that $(\eta \varphi) \partial=\partial(\eta \varphi)$. 
Passing to the direct limit of both sides as $(A, B)$ varies over closed pairs in $(U, V)$ yields the homomorphism

$$
\bar{\varphi}:{ }^{X} \Delta_{q}^{\infty}(U, V ; \operatorname{hom}(\Gamma, G)) \rightarrow \underline{\Delta}_{q}(U, V ; \operatorname{hom}(\Gamma, G))
$$

and $\bar{\varphi} \partial=\partial \bar{\varphi}$. So $\bar{\varphi}$ is a natural chain map and determines a homomorphism $\bar{\varphi}_{*}$ from ${ }^{X} H_{*}^{\infty}(\cdot, \cdot ; \operatorname{hom}(\Gamma, G)), \quad \partial_{*} \quad$ to $\underline{H}_{*}(\cdot, \cdot ; \operatorname{hom}(\Gamma, G)), \partial_{*}$ both nonnegative additive homology functors on $X$.

If $X$ is finite dimensional it would follow from Theorem 2.2 that if $\bar{\varphi}_{*}$ were an isomorphism for pairs of the form $(X, X-x)$ for $x \in X$ then $\bar{\varphi}_{*}$ would be an isomorphism for all open $(U, V)$ in $X$. To obtain the local result we consider the case of an $n$ manifold (i.e. a paracompact Hausdorff space in which each point has an open neighborhood homomorphic to $\mathbb{R}^{n}$ ).

LeMma 8.1. If $X$ is an $n$ manifold, then

$$
\bar{\varphi}_{*}:{ }^{X} H_{*}^{\infty}(X, X-x ; \operatorname{hom}(\Gamma, G)) \approx \underline{H}_{*}(X, X-x ; \operatorname{hom}(\Gamma, G))
$$

is an isomorphism for all $x \in X$.

Proof. Since $X-(X-x)=x$ is compact, it follows from Lemma 7.2 that

$$
{ }^{X} H_{q}^{\infty}(X, X-x ; \operatorname{hom}(\Gamma, G)) \approx H_{q}(X, X-x ; \operatorname{hom}(\Gamma, G)) .
$$

Let $U$ be an open neighborhood of $x$ with $\bar{U}$ homomorphic to a closed $n$ ball. Then

$$
H_{q}(\bar{U}, \bar{U}-x ; \operatorname{hom}(\Gamma, G)) \approx H_{q}(X, X-x ; \operatorname{hom}(\Gamma, G)) .
$$

Since $\bar{U}$ is simply connected, $\operatorname{hom}(\Gamma, G)$ is equivalent to a constant system on $\bar{U}$ so that

$$
H_{q}(\bar{U}, \bar{U}-x ; \operatorname{hom}(\Gamma, G)) \approx \begin{cases}0, & q \neq n, \\ \operatorname{hom}\left(\Gamma_{x}, G\right), & q \neq n .\end{cases}
$$

For the other group note that cofinal in the family of all closed pairs in $(X, X-x)$ are pairs of the form $(X, X-U)$ where $U$ is as above. Then $\bar{\Delta}_{c}^{*}(X, X-U ; \Gamma)$ and $\bar{\Delta}_{c}^{*}(\bar{U}, \bar{U}-U ; \Gamma)$ are chain equivalent. Since $\bar{U}$ is simply connected,

$$
H^{q}\left(\bar{\Delta}_{c}^{*}(\bar{U}, \bar{U}-U ; \Gamma)\right) \approx \begin{cases}0, & q \neq n \\ \Gamma_{x}, & q=n\end{cases}
$$


Therefore,

$$
\begin{aligned}
\underline{H}_{q}(X & , X-x ; \operatorname{hom}(\Gamma, G)) \\
& =H_{q}\left(\underline{\Delta}_{*}(X, X-x ; \operatorname{hom}(\Gamma, G))\right) \\
& =H_{q}\left(\varliminf_{\longrightarrow}\left\{\operatorname{hom}\left(\bar{\Delta}_{c}^{*}(X, X-U ; \Gamma), Q\right)\right\}\right) \\
& \approx \lim _{\longrightarrow}\left\{H_{q}\left(\operatorname{hom} \bar{\Delta}_{c}^{*}(X, X-U ; \Gamma), Q\right)\right\} \\
& \left.\approx \underset{\lim }{\longrightarrow} H_{q}\left(\operatorname{hom} \bar{\Delta}_{c}^{*}(\bar{U}, \bar{U}-U ; \Gamma), Q\right)\right\} \\
& \approx \begin{cases}0, & q \neq n, \\
\operatorname{hom}\left(\Gamma_{x}, G\right), & q=n,\end{cases}
\end{aligned}
$$

the last isomorphism by the universal coefficient formula.

Finally, we observe that the pairing we defined induces a pairing

$$
{ }^{X} H_{n}(\bar{U}, \bar{U}-x ; \operatorname{hom}(\Gamma, G)) \times H_{c}^{n}(\bar{U}, \bar{U}-U ; \Gamma) \rightarrow G
$$

which is isomorphic to the evaluation pairing

$$
\operatorname{hom}\left(\Gamma_{x}, G\right) \times \Gamma_{x} \rightarrow G .
$$

Therefore

$$
\bar{\varphi}_{*}:{ }^{X} H_{n}^{\infty}(X, X-x ; \operatorname{hom}(\Gamma, G)) \rightarrow \underline{H}_{n}(X-X-x ; \operatorname{hom}(\Gamma, G))
$$

is an isomorphism (corresponding to the identity map of $\operatorname{hom}\left(\Gamma_{x}, G\right)$ $\rightarrow \operatorname{hom}\left(\Gamma_{x}, G\right)$ resulting from the evaluation pairing above).

TheOREM 8.2. For an $n$ manifold $X$

$$
\bar{\varphi}_{*}:{ }^{X} H_{*}^{\infty}(U, V ; \operatorname{hom}(\Gamma, G)) \approx \underline{H}_{*}(U, V ; \operatorname{hom}(\Gamma, G))
$$

for every open pair $(U, V)$ in $X$.

Proof. Using Lemma 8.1 the result follows immediately from Theorem 2.2

From the definitions we see that if $(A, B)$ is closed in $X$ then $\underline{H}_{*}(A, B ; \operatorname{hom}(\Gamma, G))=H_{*}\left(\operatorname{hom}\left(\bar{\Delta}_{c}^{*}(A, B ; \Gamma), Q\right)\right)$. We already remarked that if $X$ is HLC then, $\bar{\Delta}_{c}^{*}(A, B ; \Gamma)$ has cohomology isomorphic to the Čech-Alexander cohomology of $(A, B)$ with compact supports. It follows that in a locally compact HLC space $X$ the groups $\underline{H}_{*}(A, B ; \operatorname{hom}(\Gamma, G))$ are isomorphic to the Borel-Moore homology groups [1] of $(A, B)$ with coefficients $\operatorname{hom}(\Gamma, G)$. 
9. Homology-cohomology comparison. In this section we construct a homomorphism from singular homology ${ }^{X} H_{*}^{\infty}(U, V)$ to singular cohomology $H^{*}(X-V, X-U)$ with suitable coefficient systems. This homomorphism will depend on a cohomology class of $H^{*}(X \times X, X \times X-\delta(X))$ where $\delta(X)=\{(x, y) \in X \times X \mid x=y\}$ is the diagonal of $X \times X$.

We shall assume $\mathscr{V}$ is an open covering of $X$ such that if $V$, $V^{\prime} \in \mathscr{V}$ and $V \cap V^{\prime} \neq \varnothing$ then every closed path in $V \cup V^{\prime}$ is null homotopic in $X$. If $X$ is a paracompact space in which each point has an open neighborhood $W$ with the property that every closed path in $W$ is null homotopic in $X$, such a covering can be obtained as follows. Let $\mathscr{W}$ be an open covering of $X$ by sets $W$ such that every closed path in $W$ is null homotopic in $X$ (W $\mathscr{W}$ is assumed to exist) and let $\mathscr{V}$ be an open star refinement of $\mathscr{W}$ covering $X$ (such star refinements exist because $X$ is paracompact). Then $\mathscr{V}$ has the desired property.

In $\S \S 6$ and 7 it was noted that for any $A \subset X$ the inclusion maps

$$
{ }^{X} \Delta_{*}^{\infty}(\mathscr{V} \cap A ; \Gamma) \subset{ }^{X} \Delta_{*}^{\infty}(A ; \Gamma), \quad \Delta_{*}(\mathscr{V} \cap A ; \Gamma) \subset \Delta_{*}(A ; \Gamma)
$$

are chain equivalences (where $\mathscr{V} \cap A=\{V \cap A \mid V \in \mathscr{V}\}$ ). If $\sigma$ and $\sigma^{\prime}$ are singular simplexes such that $|\sigma| \subset V,\left|\sigma^{\prime}\right| \subset V^{\prime}$ for $V, V^{\prime} \in \mathscr{V}$ and $|\sigma| \cap\left|\sigma^{\prime}\right| \neq \varnothing$ then if $x \in|\sigma| \cap\left|\sigma^{\prime}\right|$ there are isomorphisms

$$
\Gamma(\sigma) \underset{\approx}{\approx} \underset{\approx}{\approx} \Gamma\left(\sigma^{\prime}\right)
$$

If $x^{\prime}$ is another point in $|\sigma| \cap\left|\sigma^{\prime}\right|$ let $\omega$ be a path in $|\sigma|$ from $x$ to $x^{\prime}$ and $\omega^{\prime}$ a path in $\left|\sigma^{\prime}\right|$ from $x$ to $x^{\prime}$. There are commutative triangles

$$
\begin{gathered}
\Gamma(\sigma) \underset{\Gamma_{x^{\prime}}}{\approx} \approx \Gamma_{x} \\
\end{gathered}
$$

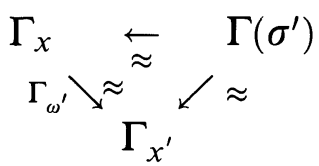

Since $\omega, \omega^{\prime}$ have the same endpoints and lie in $V \cup V^{\prime}$ they are homotopic in $X$ so $\Gamma_{\omega}=\Gamma_{\omega^{\prime}}: \Gamma_{x} \rightarrow \Gamma_{x^{\prime}}$. It follows that the composite of the isomorphisms

$$
\Gamma(\sigma) \underset{\approx}{\longrightarrow} \Gamma_{x} \overleftarrow{\approx} \Gamma\left(\sigma^{\prime}\right)
$$

is independent of the choice of $x \in|\sigma| \cap\left|\sigma^{\prime}\right|$ so there is a well-defined isomorphism $\lambda_{\sigma \sigma^{\prime}}: \Gamma(\sigma) \approx \Gamma\left(\sigma^{\prime}\right)$.

If $\sigma, \sigma^{\prime}, \sigma^{\prime \prime}$ are singular simplexes such that $|\sigma| \subset V,\left|\sigma^{\prime}\right| \subset V^{\prime}$, $\left|\sigma^{\prime \prime}\right| \subset V^{\prime \prime}$ for $V, V^{\prime}, V^{\prime \prime} \in \mathscr{V}$ and $|\sigma| \cap\left|\sigma^{\prime}\right|,|\sigma| \cap\left|\sigma^{\prime \prime}\right|,\left|\sigma^{\prime}\right| \cap\left|\sigma^{\prime \prime}\right|$ are all non-empty, then $\lambda_{\sigma \sigma^{\prime \prime}}=\lambda_{\sigma^{\prime} \sigma^{\prime \prime}} \lambda_{\sigma \sigma^{\prime}}: \Gamma(\sigma) \approx \Gamma\left(\sigma^{\prime \prime}\right)$. 
Using this notation if $g \in \Gamma(\sigma)$, then $g \mid \sigma^{(j)}=\lambda_{\sigma \sigma(j)}(g)$. It follows that if $c=\sum_{\sigma} g_{\sigma} \sigma \in \Delta_{*}^{\infty}(\mathscr{V} ; \Gamma)$, then

$$
\partial c=\sum_{\sigma} \sum_{j}(-1)^{j} \lambda_{\sigma \sigma(j)}\left(g_{\sigma}\right) \sigma^{(j)}
$$

and if $u \in \Delta^{q}(\mathscr{V} ; \Gamma)$, then

$$
(\delta u)(\sigma)=\sum_{i}(-1)^{i} \lambda_{\sigma^{(i)} \sigma}\left(u\left(\sigma^{(i)}\right)\right)
$$

If $\Gamma$ and $\Gamma^{\prime}$ are local systems on $X$ define $\Gamma \times \Gamma^{\prime}$ to be the local system on $X \times X$ such that $\left(\Gamma \times \Gamma^{\prime}\right)_{(x, y)}=\Gamma_{x} \otimes \Gamma_{y}^{\prime}$ and $\left(\Gamma \times \Gamma^{\prime}\right)_{\omega}=$ $\Gamma_{\mathrm{pr}_{1} \omega} \otimes \Gamma_{\mathrm{pr}_{2} \omega}$ where $\mathrm{pr}_{1}: X \times X \rightarrow X$ and $\mathrm{pr}_{2}: X \times X \rightarrow X$ are projections to the first and second coordinates, respectively.

Let $U \in H^{n}(X \times X, X \times X-\delta(X)$; $\operatorname{hom}(R \times \Gamma, R))$ be a given cohomology class (here we regard $R$ as a constant local system on $X$ in forming $R \times \Gamma$ on $X \times X)$ and let

$$
u \in \operatorname{hom}\left(\Delta_{n}(X \times X, X \times X-\delta(X) ; R \times \Gamma), R\right)
$$

be a cocycle representing $U$.

Let $\tau: \Delta_{*}(X ; R) \otimes \Delta_{*}(X ; \Gamma) \rightarrow \Delta_{*}(X \times X, R \times \Gamma)$ be an Eilenberg-Zilber map [8]. Then $\tau$ is a natural chain map (so $\tau\left(\Delta_{*}(Y ; R) \otimes \Delta_{*}(Z ; \Gamma)\right) \subset \Delta_{*}(Y \times Z, R \times \Gamma)$ for all $\left.Y, Z \subset X\right)$, and in dimension 0 , if $[x]$ denotes the 0 -singular simplex at the point $x$, then

$$
\tau(\alpha[x] \otimes g[y])=\alpha g[(x, y)]
$$

for $\alpha \in R, g \in \Gamma_{y}$ and $x, y \in X$. For an arbitrary local system $\Gamma^{\prime}$ on $X$ define

$$
\theta:{ }^{X} \Delta_{q}^{\infty}\left(\mathscr{V} ; \Gamma \otimes \Gamma^{\prime}\right) \rightarrow \Delta^{n-q}\left(\mathscr{V} ; \Gamma^{\prime}\right)
$$

by requiring it to be $R$ linear and such that if $\sigma^{\prime}$ is an $(n-q)$ singular simplex in $V^{\prime}$ where $V^{\prime} \in \mathscr{V}$ and $c \in{ }^{X} \Delta_{q}^{\infty}\left(\mathscr{V} \cap(X-|\sigma|) ; \Gamma \otimes \Gamma^{\prime}\right)$ then

$$
\theta(c)\left(\sigma^{\prime}\right)=0
$$

while if $c=\left(g \otimes g^{\prime}\right) \sigma \in \Delta_{q}\left(\mathscr{V}, \Gamma \otimes \Gamma^{\prime}\right)$ then

$$
\theta(c)\left(\sigma^{\prime}\right)=\left\langle u, \tau\left(\sigma^{\prime} \otimes g \sigma\right)\right\rangle \lambda_{\sigma \sigma^{\prime}}\left(g^{\prime}\right)=\lambda_{\sigma \sigma^{\prime}}\left(\left\langle u, \tau\left(\sigma^{\prime} \otimes g \sigma\right)\right\rangle g^{\prime}\right)
$$

(the right-hand side is 0 if $|\sigma| \cap\left|\sigma^{\prime}\right|=\varnothing$ because, in that case

$$
\tau\left(\sigma^{\prime} \otimes g \sigma\right) \subset X \times X-\delta(X)
$$

and $u$ vanishes on $\left.\Delta_{n}(X \times X, X \times X-\delta(X) ; R \times \Gamma)\right)$. This uniquely 
defines $\theta$ because, for every singular simplex $\sigma^{\prime}$, given $c \in$ ${ }^{x} \Delta_{q}^{\infty}\left(\mathscr{V} ; \Gamma \otimes \Gamma^{\prime}\right)$ there exist $c_{1} \in \Delta_{q}\left(\mathscr{V} ; \Gamma \otimes \Gamma^{\prime}\right)$ and $c_{2} \in$ ${ }^{X} \Delta_{q}^{\infty}\left(\mathscr{V} \cap\left(X-\left|\sigma^{\prime}\right|\right) ; \Gamma \otimes \Gamma\right)$ such that $c=c_{1}+c_{2}$ (so that $\theta\left(c_{2}\right)\left(\sigma^{\prime}\right)=0$ and $\theta\left(c_{1}\right)\left(\sigma^{\prime}\right)$ is a finite sum of terms of the form described above).

Given $\sigma^{\prime}$ in $\Delta_{n-q+1}\left(V^{\prime}\right)$ for some $V^{\prime} \in \mathscr{V}$ let $c=c_{1}+c_{2}$ where $c_{1} \in \Delta_{q}\left(\mathscr{V} ; \Gamma \otimes \Gamma^{\prime}\right)$ and $c_{2} \in{ }^{X} \Delta_{q}^{\infty}\left(\mathscr{V} \cap\left(X-\left|\sigma^{\prime}\right|\right) ; \Gamma \otimes \Gamma^{\prime}\right)$. Then $\delta(\theta(c))=\delta\left(\theta\left(c_{1}\right)\right)+\delta\left(\theta\left(c_{2}\right)\right)$ and $\left(\delta\left(\theta\left(c_{2}\right)\right)\right)\left(\sigma^{\prime}\right)=0$ so $(\delta(\theta(c)))\left(\sigma^{\prime}\right)$ $=\left(\delta\left(\theta\left(c_{1}\right)\right)\right)\left(\sigma^{\prime}\right)$. To calculate $\left(\delta\left(\theta\left(c_{1}\right)\right)\right)\left(\sigma^{\prime}\right)$ we need only calculate it for $c_{1}$ of the form $\left(g \otimes g^{\prime}\right) \sigma$ where $|\sigma| \cap\left|\sigma^{\prime}\right| \neq \varnothing$. In this case

$$
\begin{aligned}
\left(\delta \theta\left(\left(g \otimes g^{\prime}\right) \sigma\right)\right)\left(\sigma^{\prime}\right) & =\sum_{i}(-1)^{i} \lambda_{\sigma^{\prime(i)} \sigma^{\prime}}\left[\theta\left(\left(g \otimes g^{\prime}\right) \sigma\right)\left(\sigma^{\prime(i)}\right)\right] \\
& =\sum_{i}(-1)^{i} \lambda_{\sigma^{\prime(i)} \sigma^{\prime}}\left[\left\langle u, \tau\left(\sigma^{\prime(i)} \otimes g \sigma\right)\right\rangle \lambda_{\sigma \sigma^{\prime(i)}}\left(g^{\prime}\right)\right]
\end{aligned}
$$

where the corresponding term on the right is 0 if $|\sigma| \cap\left|\sigma^{\prime(i)}\right|=\varnothing$. If $|\sigma| \cap\left|\sigma^{\prime(i)}\right| \neq \varnothing$, then $\lambda_{\sigma^{\prime(i)} \sigma^{\prime}} \lambda_{\sigma \sigma^{\prime(i)}}=\lambda_{\sigma \sigma^{\prime}}$ so we obtain

$$
\sum_{i}(-1)^{i}\left\langle u, \tau\left(\sigma^{\prime(i)} \otimes g \sigma\right)\right\rangle \lambda_{\sigma \sigma^{\prime}}\left(g^{\prime}\right)=\left\langle u, \tau\left(\partial \sigma^{\prime} \otimes g \sigma\right)\right\rangle \lambda_{\sigma \sigma^{\prime}}\left(g^{\prime}\right) .
$$

Now $\partial \tau\left(\sigma^{\prime} \otimes g \sigma\right)=\tau\left(\partial \sigma^{\prime} \otimes g \sigma\right)+(-1)^{n-q+1} \tau\left(\sigma^{\prime} \otimes \partial(g \sigma)\right)$ so that $\tau\left(\partial \sigma^{\prime} \otimes g \sigma\right)=\partial \tau\left(\sigma^{\prime} \otimes g \sigma\right)+(-1)^{n-q} \tau\left(\sigma^{\prime} \otimes \partial(g \sigma)\right)$, and we obtain

$$
\begin{aligned}
\langle u, \partial \tau & \left.\left(\sigma^{\prime} \otimes g \sigma\right)\right\rangle \lambda_{\sigma \sigma^{\prime}}\left(g^{\prime}\right)+(-1)^{n-q}\left\langle u, \tau\left(\sigma^{\prime} \otimes \partial(g \sigma)\right)\right\rangle \lambda_{\sigma \sigma^{\prime}}\left(g^{\prime}\right) \\
= & \left\langle\delta u, \tau\left(\sigma^{\prime} \otimes g \sigma\right)\right\rangle \lambda_{\sigma \sigma^{\prime}}\left(g^{\prime}\right) \\
& +(-1)^{n-q} \sum_{j}(-1)^{j}\left\langle u, \tau\left(\sigma^{\prime} \otimes \lambda_{\sigma \sigma^{(j)}}(g) \sigma^{(j)}\right)\right\rangle \lambda_{\sigma \sigma^{\prime}}\left(g^{\prime}\right) .
\end{aligned}
$$

Because $\delta u=0$ since $u$ is a cocycle, this equals

$$
(-1)^{n-q} \sum_{j}(-1)^{j}\left\langle u, \tau\left(\sigma^{\prime} \otimes \lambda_{\sigma \sigma^{(j)}}(g) \sigma^{(j)}\right)\right\rangle \lambda_{\sigma \sigma^{\prime}}\left(g^{\prime}\right) .
$$

The corresponding term on the right equals 0 if $\left|\sigma^{(j)}\right| \cap\left|\sigma^{\prime}\right|=\varnothing$ and if $\left|\sigma^{(j)}\right| \cap\left|\sigma^{\prime}\right| \neq \varnothing$, then $\lambda_{\sigma \sigma^{\prime}}=\lambda_{\sigma^{(j)} \sigma^{\prime}} \lambda_{\sigma \sigma^{(j)}}$ so we obtain

$$
\begin{aligned}
& (-1)^{n-q} \sum_{j}(-1)^{j}\left\langle u, \tau\left(\sigma^{\prime} \otimes \lambda_{\sigma \sigma^{(j)}}(g) \sigma^{(j)}\right)\right\rangle \lambda_{\sigma^{(\jmath)} \sigma^{\prime}} \lambda_{\sigma \sigma^{(j)}}\left(g^{\prime}\right) \\
& =(-1)^{n-q} \sum_{j}(-1)^{j} \lambda_{\sigma^{\prime} \sigma^{\prime}}\left[\left\langle u, \tau\left(\sigma^{\prime} \otimes \lambda_{\sigma \sigma^{(j)}}(g) \sigma^{(j)}\right)\right\rangle \lambda_{\sigma \sigma^{(j)}}\left(g^{\prime}\right)\right]
\end{aligned}
$$

and, by definition, this equals $(-1)^{n-q} \theta\left(\partial\left(g \otimes g^{\prime}\right) \sigma\right)\left(\sigma^{\prime}\right)$. Therefore, $\theta$ maps the chain complex ${ }^{X} \Delta_{*}^{\infty}\left(\mathscr{V} ; \Gamma \otimes \Gamma^{\prime}\right)$ into the cochain complex $\Delta^{*}\left(\mathscr{V} ; \Gamma^{\prime}\right)$ so that it commutes up to sign with $\partial$ and $\delta$. 
If $B \subset X$ is arbitrary, then

$$
\tau\left(\Delta_{*}(\mathscr{V} \cap(X-B) ; R) \otimes \Delta_{*}(\mathscr{V} \cap B ; \Gamma)\right) \subset \Delta_{*}((X-B) \times B ; R \times \Gamma)
$$

and $u$ vanishes on the latter. Therefore, $\theta$ maps

$$
{ }^{X} \Delta_{*}^{\infty}\left(\mathscr{V} \cap(X-B) ; \Gamma \otimes \Gamma^{\prime}\right) \text { into } \Delta^{*}\left(\mathscr{V}, \mathscr{V} \cap B ; \Gamma^{\prime}\right) .
$$

If $B \subset A \subset X$, the following diagram is commutative (the top row is a short exact sequence with coefficients $\Gamma \otimes \Gamma^{\prime}$, the bottom row is a short exact sequence with coefficients $\Gamma^{\prime}$, and the right-hand vertical map is defined to make the diagram commutative)

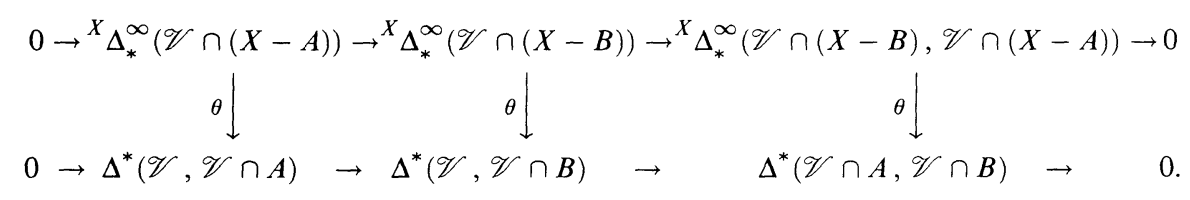

If $\left(A^{\prime}, B^{\prime}\right) \subset(A, B)$ there is a commutative square

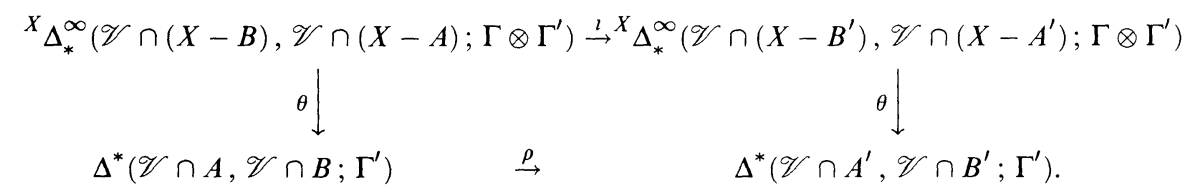

Let $(A, B)$ be a closed pair. Taking the direct limit of the homomorphisms (as $(V, W)$ varies over open neighborhoods of $(A, B))$

$\theta:{ }^{X} \Delta_{*}^{\infty}\left(\mathscr{V} \cap(X-W), \mathscr{V} \cap(X-V) ; \Gamma \otimes \Gamma^{\prime}\right) \rightarrow \Delta^{*}\left(\mathscr{V} \cap V, \mathscr{V} \cap W ; \Gamma^{\prime}\right)$

determines the homomorphism

$\bar{\theta}:{ }^{X} \Delta_{*}^{\infty}\left(\mathscr{V} \cap(X-B), \mathscr{V} \cap(X-A) ; \Gamma \otimes \Gamma^{\prime}\right) \rightarrow \bar{\Delta}^{*}\left(\mathscr{V} \cap A, \mathscr{V} \cap B ; \Gamma^{\prime}\right)$.

Similarly taking the direct limit as $(V, W)$ varies over open cobounded neighborhoods of $(A, B)$ in $X$ (and observing that, in case $(V, W)$ is cobounded,

$$
\begin{aligned}
& { }^{X} \Delta_{*}^{\infty}\left(\mathscr{V} \cap(X-W), \mathscr{V} \cap(X-V) ; \Gamma \otimes \Gamma^{\prime}\right) \\
& \left.\quad=\Delta_{*}\left(\mathscr{V} \cap(X-W), \mathscr{V} \cap(X-V) ; \Gamma \otimes \Gamma^{\prime}\right)\right)
\end{aligned}
$$

we obtain a homomorphism

$\left.\bar{\theta}^{c}: \Delta_{*}\left(\mathscr{V} \cap(X-B), \mathscr{V} \cap(X-A) ; \Gamma \otimes \Gamma^{\prime}\right) \rightarrow \bar{\Delta}_{\mathcal{C}}^{*}\left(\mathscr{V} \cap A, \mathscr{V} \cap B ; \Gamma^{\prime}\right)\right)$.

The homomorphisms $\bar{\theta}$ and $\bar{\theta}^{c}$ depend on the choice of the cocycle $u$ and the Eilenberg-Zilber map $\tau$. Altering $u$ in the cohomology class 
$U$ or altering $\tau$ will alter $\bar{\theta}$ and $\bar{\theta}^{c}$ by a chain homotopy. Therefore,

$\bar{\theta}$ induces uniquely defined homomorphisms

$$
\begin{aligned}
\bar{\theta}_{U}:{ }^{X} H_{q}^{\infty}(\mathscr{V} \cap(X-B), \mathscr{V} \cap(X-A) & \left.; \Gamma \otimes \Gamma^{\prime}\right) \\
& \rightarrow \bar{H}^{n-q}\left(\mathscr{V} \cap A, \mathscr{V} \cap B ; \Gamma^{\prime}\right)
\end{aligned}
$$

and

$$
\begin{aligned}
\bar{\theta}_{U}^{c}: H_{q}(\mathscr{V} \cap(X-B), \mathscr{V} \cap(X-A) & \left.; \Gamma \otimes \Gamma^{\prime}\right) \\
& \rightarrow \bar{H}_{c}^{n-q}\left(\mathscr{V} \cap A, \mathscr{V} \cap B ; \Gamma^{\prime}\right) .
\end{aligned}
$$

The homomorphism

$$
\bar{\theta}_{U}:{ }^{X} H_{q}^{\infty}\left(X-B, X-A ; \Gamma \otimes \Gamma^{\prime}\right) \rightarrow \bar{H}^{n-q}\left(A, B ; \Gamma^{\prime}\right)
$$

is defined so that commutativity holds in the square

$$
\begin{array}{ccc}
{ }^{X} H_{q}^{\infty}\left(\mathscr{V} \cap(X-B), \mathscr{V} \cap(X-A) ; \Gamma \otimes \Gamma^{\prime}\right) & \approx & { }^{X} H_{q}^{\infty}\left(X-B, X-A ; \Gamma \otimes \Gamma^{\prime}\right) \\
\bar{\theta}_{U} \downarrow & & \downarrow^{\bar{\theta}_{U}} \\
\bar{H}^{n-q}\left(\mathscr{V} \cap A, \mathscr{V} \cap B ; \Gamma^{\prime}\right) & \longleftarrow & \bar{H}^{n-q}\left(A, B ; \Gamma^{\prime}\right) .
\end{array}
$$

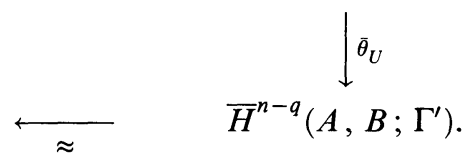

Similarly the homomorphism

$$
\bar{\theta}_{U}^{c}: H_{q}\left(X-B, X-A ; \Gamma \otimes \Gamma^{\prime}\right) \rightarrow \bar{H}_{c}^{n-q}\left(A, B ; \Gamma^{\prime}\right)
$$

is defined so that commutativity holds in the square

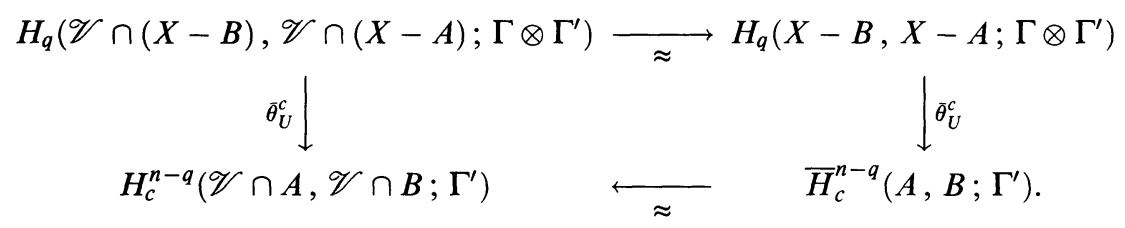

Both $\bar{\theta}_{U}$ and $\bar{\theta}_{U}^{c}$ are natural and commute up to sign with connecting homomorphisms. The image $\bar{\theta}_{U}(z)$ is the slant product $U / z$ $[7,8]$ for local coefficients.

10. Duality in manifolds. Throughout this section $X$ will be assumed to be an $n$ manifold. We prove various duality theorems relating homology of a pair in $X$ to cohomology of the complementary. pair.

TheOREM 10.1. Suppose $X=\bigcup_{i=0}^{\infty}$ int $C_{i}$ where $\bar{C}_{i}$ is compact and $C_{i} \subset C_{i+1}$ for each $i$. Then for any local system on $X$,

$$
H_{n}^{\infty}(X ; \Gamma) \approx \lim \left\{H_{n}\left(X, X-C_{i}, \Gamma\right)\right\}
$$


Proof. By Lemma 1 on p. 299 of [8] $H_{n+1}\left(X, X-C_{i}, \Gamma\right)=0$ for each $i$ (the lemma referred to asserts the result for a constant local system $G$, but the same argument establishes the result for an arbitrary local system $\Gamma$ ). The theorem follows from this using Theorem 7.3.

It is straightforward to verify that $\lim \left\{H_{n}\left(X, X-C_{i} ; G\right)\right\}$ is the same as $H_{n}^{c}(X ; G)$ as defined on p. 299 of [8]. From Theorem 6 on p. 303 of [8] it follows that for a connected $n$ manifold $X, H_{n}^{\infty}(X ; R) \neq$ 0 if and only if $X$ is orientable over $R$, and from Theorem 5 on p. 302 of [8] there is a bijection between orientations of $X$ over $R$ and generators of $H_{n}^{\infty}(X ; R)$.

In general there is a local system $\Gamma^{X}$ on $X$ with

$$
\Gamma_{x}^{X}=H^{n}(X, X-x ; R)
$$

and, if $\omega$ is a path in $X, \Gamma_{\omega}^{X}: \Gamma_{\omega(0)}^{X} \rightarrow \Gamma_{\omega(1)}^{X}$ is suitably defined as in [7] by "moving along $\omega$ ". The dual local system $\Gamma^{X_{*}}=\operatorname{hom}\left(\Gamma^{X}, R\right)$ has the property that $\Gamma_{x}^{X_{*}} \approx H_{n}(X, X-x ; R)$ for all $x \in X$. In fact, $\Gamma_{x}^{X} \approx R$ for each $x \in X$ and $\Gamma_{\omega}^{X}$ corresponds to multiplication of $R$ by \pm 1 depending on $\omega$. Therefore, $\Gamma^{X_{*}} \approx \Gamma^{X}$. On $X \times X$ the local system $R \times \Gamma^{X_{*}}$ is isomorphic to $\operatorname{hom}\left(R \times \Gamma^{X}, R\right)$.

For $x \in X$ there are isomorphisms

$$
\begin{aligned}
H_{n}\left(X, X-x ; \Gamma^{X}\right) & \approx \operatorname{hom}\left(H^{n}(X, X-x ; R) ; \Gamma_{x}^{X}\right) \\
& \approx \operatorname{hom}\left(H^{n}(X, X-x ; R), H^{n}(X, X-x ; R)\right)
\end{aligned}
$$

and $z_{x} \in H_{n}\left(X, X-x ; \Gamma^{X}\right)$ will denote the element corresponding under the above to the identity map of $H^{n}(X, X-x ; R)$.

A Thom class on $X$ is an element

$$
U \in H^{n}\left(X \times X, X \times X-\delta(X) ; \operatorname{hom}\left(R \times \Gamma^{X}, R\right)\right)
$$

such that, for each $x \in X, \bar{\theta}_{U}^{c}\left(z_{x}\right)=1 \in \bar{H}_{c}^{0}(x ; R)$. It is known (Theorem 4.7 in [7]) that every manifold has a unique Thom class. In the sequel we use the Thom class $U$ in defining $\bar{\theta}$ and $\bar{\theta}^{c}$ and omit specific reference to $U$ in the notation.

THEOREM 10.2. For every closed pair $(A, B)$ in $X$ and every local system $\Gamma$ of $R$ modules on $X$ there are isomorphisms

$$
\bar{\theta}:{ }^{X} H_{q}^{\infty}\left(X-B, X-A ; \Gamma^{X} \otimes \Gamma\right) \approx \bar{H}^{n-q}(A, B ; \Gamma)
$$


and

$$
\bar{\theta}^{c}: H_{q}\left(X-B, X-A ; \Gamma^{X} \otimes \Gamma\right) \approx \bar{H}_{c}^{n-q}(A, B ; \Gamma) .
$$

Proof. Define cohomology theories $H^{\prime}$ and $\bar{H}$ on $X$ by $H^{\prime j}(A, B)$ $={ }^{X} H_{n-j}^{\infty}\left(X-B, X-A ; \Gamma^{X} \otimes \Gamma\right)$ and $\bar{H}^{j}(A, B)=\bar{H}^{j}(A, B ; \Gamma)$ with $\delta^{\prime}, \bar{\delta}$ suitably defined connecting homomorphisms. Then $\bar{\theta}$ is a homomorphism of $H^{\prime}, \delta^{\prime}$ into $\bar{H}, \bar{\delta}$ which is an isomorphism for every $x \in X$ (because $H^{j}(x)={ }^{X} H_{n-j}^{\infty}\left(X, X-x ; \Gamma^{X} \otimes \Gamma\right)=$ $H_{n-j}\left(X, X-x ; \Gamma^{X} \otimes \Gamma\right) \approx H_{n-j}(X, X-x ; R) \otimes \Gamma_{x} \bar{H}^{j}(x)=$ $\bar{H}^{j}(x ; \Gamma) \approx \bar{H}^{j}(x ; R) \otimes \Gamma_{x}$, and both sides are 0 except for $j=: 0$ when $\bar{\theta}$ is an isomorphism by the choice of $U$ ). Since $H^{\prime}$ and $\bar{H}$ are additive and $X$ is finite dimensional, it follows from Theorem 2.1 that $\bar{\theta}$ is an isomorphism for all closed $(A, B)$ in $X$.

The result for $\bar{\theta}^{c}$ is obtained similarly because the two sides being compared are weakly additive cohomology functors and $\bar{\theta}^{c}$ is an isomorphism for every $x \in X$.

REMARK 10.3. Replacing $\Gamma$ by $\Gamma^{X} \otimes \Gamma$ and noting that $\Gamma^{X} \otimes \Gamma^{X} \approx R$ so that $\Gamma^{X} \otimes\left(\Gamma^{X} \otimes \Gamma\right) \approx R \otimes \Gamma \approx \Gamma$ we see that for $(A, B)$ closed in $X$ there are also isomorphisms

$$
\bar{\theta}:{ }^{X} H_{q}^{\infty}(X-B, X-A ; \Gamma) \approx \bar{H}^{n-q}\left(A, B ; \Gamma^{X} \otimes \Gamma\right)
$$

and

$$
\bar{\theta}^{c}: H_{q}(X-B, X-A ; \Gamma) \approx \bar{H}_{c}^{n-q}\left(A, B ; \Gamma^{X} \otimes \Gamma\right)
$$

for an arbitrary local system $\Gamma$.

Theorem 10.2 and Remark 10.3 express duality between the two types of singular homology groups of an open pair in $X$ (i.e. weakly additive or additive homology) with two types of Čech-Alexander cohomology groups of the complementary closed pair (either weakly additive or additive cohomology) with arbitrary coefficient systems. This duality is not just an isomorphism of the homology groups with cohomology groups but is an isomorphism of cohomology theories with all its implications. There is also the following result which expresses duality between the Borel-Moore homology of a closed pair in $X$ and the singular cohomology of the complementary open pair (which equals the Čech-Alexander cohomology) of the open pair.

THEOREM 10.4. If $(A, B)$ is a closed pair in $X$ and $\Gamma$ is a local system on $X$ there is an isomorphism

$$
\underline{H}_{n-q}(A, B ; \operatorname{hom}(\Gamma, G)) \approx H^{q}\left(X-B, X-A ; \Gamma^{X} \otimes \operatorname{hom}(\Gamma, G)\right) .
$$


Proof. In $\S 9$ we defined a map

$\bar{\theta}^{c}: \Delta_{*}\left(\mathscr{V} \cap(X-B), \mathscr{V} \cap(X-A) ; \Gamma^{X} \otimes \Gamma\right) \rightarrow \bar{\Delta}_{c}^{*}(\mathscr{V} \cap A, \mathscr{V} \cap B ; \Gamma)$

for $(A, B)$ a closed pair in $X$. Define a chain complex $(C, \partial)$ so that $C_{q}=\bar{\Delta}_{c}^{n-q}(\mathscr{V} \cap A, \mathscr{V} \cap B ; \Gamma)$ and $\partial: C_{q} \rightarrow C_{q-1}$ equals $\delta:$ $\bar{\Delta}_{c}^{n-q}(\mathscr{V} \cap A, \mathscr{V} \cap B ; \Gamma) \rightarrow \bar{\Delta}_{c}^{n-q+1}(\mathscr{V} \cap A, \mathscr{V} \cap B ; \Gamma)$. Then $\bar{\theta}^{c}$ is a map of degree 0 from $\Delta_{*}\left(\mathscr{V} \cap(X-B), \mathscr{V} \cap(X-A) ; \Gamma^{X} \otimes \Gamma\right)$ to $C_{*}$ which commutes up to sign with $\partial$. By Theorem 10.2, $\bar{\theta}^{c}$ induces an isomorphism on homology. It follows that $\bar{\theta}^{c}$ also induces an isomorphism on cohomology

$$
H^{*}\left(C_{*} ; G\right) \approx H^{*}\left(\Delta_{*}\left(\mathscr{V} \cap(X-B), \mathscr{V} \cap(X-A) ; \Gamma^{X} \otimes \Gamma\right) ; G\right)
$$

for any $R$ module $G$. Because of the way $C_{*}$ is defined, this yields an isomorphism

$$
\begin{aligned}
& \underline{H}_{n-q}(\mathscr{V} \cap A, \mathscr{V} \cap B ; \operatorname{hom}(\Gamma, G)) \\
& \quad \approx H^{q}\left(\mathscr{V} \cap(X-B), \mathscr{V} \cap(X-A) ; \operatorname{hom}\left(\Gamma^{X} \otimes \Gamma, G\right)\right)
\end{aligned}
$$

and this corresponds to an isomorphism

$$
\underline{H}_{n-q}(A, B ; \operatorname{hom}(\Gamma, G)) \approx H^{q}\left(X-B, X-A ; \operatorname{hom}\left(\Gamma^{X} \otimes \Gamma, G\right)\right) .
$$

Because of the special nature of the local system $\Gamma^{X}$ it is easy to see that $\operatorname{hom}\left(\Gamma^{X} \otimes \Gamma, G\right) \approx \Gamma^{X} \otimes \operatorname{hom}(\Gamma, G)$ so that

$$
\underline{H}_{n-q}(A, B ; \operatorname{hom}(\Gamma, G)) \approx H^{q}\left(X-B, X-A ; \Gamma^{X} \otimes \operatorname{hom}(\Gamma, G)\right) .
$$

In case $X$ is orientable, $\Gamma^{X} \approx R$ and the Theorems 10.2 and 10.4 assert isomorphisms of homology with coefficients in a constant system with cohomology in the same constant system. In the non-orientable case, however, if the homology is in a constant local system the corresponding cohomology has coefficients in a non-constant local system and vice versa.

\section{REFERENCES}

[1] A. Borel and J. Moore, Homology theory for locally compact spaces, Michigan Math. J., 7 (1960), 137-159.

[2] H. Cartan, Séminaire de topologie algébrique, ENS, (1948-49).

[3] A. Dold, Zur Homotopietheorie der Kettenkomplexe, Math. Annalen, 140 (1960), 278-298.

[4] J. Dugundji, Topology, Allyn and Bacon, Boston, Mass., 1968.

[5] S. Eilenberg, Singular homology theory, Ann. of Math., 45 (1944), 407-447. 
[6] W. S. Massey, Homology and Cohomology Theory, Marcel Dekker, New York, 1978.

[7] E Spanier, Duality in topological manifolds, in Colloque de Topologie Tenu a Bruxelles (Centre Belge de Recherche Mathèmatiques) (1966), 91-111.

[8] _ Algebraic Topology, Springer-Verlag, New York, New York, 1982.

[9] — Cohomology isomorphisms, Contemp. Math., 12 (1982), 315-329.

[10] __ Cohomology with supports, Pacific J. Math., 123 (1986), 447-464.

[11] Cohomology theories on compact and locally compact spaces, Revisita Matemática Iberoamericana, 2 (1986), 29-53.

[12] _ Cohomology theories on spaces, Trans. Amer. Math. Soc., 301 (1987), 149-161.

[13] - Weakly additive cohomology, Publicaciones Matemátiques, 34 (1990), 145-150.

[14] N. E. Steenrod, Homology with local coefficients, Ann. of Math., 44 (1943), 610-627.

Received August 27, 1990 and in revised form November 28, 1990.

UNIVERSITY OF CALIFORNIA

BERKELEY, CA 94720 


\section{CONTENTS}

G. D. Anderson, M. K. Vamanamurthy, and M. Vuorinen, Inequalities for quasi-

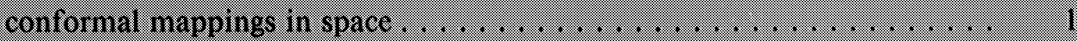

T. Bhattacharya, A nonexistence result for the $n$-Laplacian . . . . . . . . . . . . 19

J. A. Cima, K. Stroethoff, and K. Yale, Bourgain algebras on the unit disk . . . . . 27

J. A. Fridy and C. Orhan, Lacunary statistical convergence . . . . . . . . . . . . . . 43

D. Grenier, On the shape of fundamental domains in $\mathrm{GL}(n, \mathbf{R}) / \mathrm{O}(n) \ldots \ldots . . .53$

B. Jiang and J. Guo, Fixed points of surface diffeomorphisms . . . . . . . . . . . 67

P. Lejarraga, The moduli of rational Weierstrass fibrations over $\mathbf{P}^{\prime}$ : singularities 91

G. J. Martin, On discrete isometry groups of negative curvature . . . . . . . . . . 109

T. Nakashima, Adjoint linear systems on a surface of general type in positive characteristic . . . . . . . . . . . . . . . . . . . . . . . . . 129

B. Ralph, A homotopy transfer for finite group actions $\ldots \ldots \ldots \ldots \ldots \ldots \ldots$

Y. Rong, Maps between Seifert fibered spaces of infinite $\pi_{1} \ldots \ldots \ldots \ldots \ldots, 143$

J.-Y. Shi, Some numeric results on root systems . . . . . . . . . . . . 155

E. Spanier, Singular homology and cohomology with local coefficients and duality

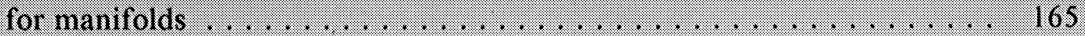




\section{PACIFIC JOURNAL OF MATHEMATICS}

Volume $160 \quad$ No. $1 \quad$ September 1993

Inequalities for quasiconformal mappings in space

GLEN DOUglas ANDERson, MaVina KRISHNa VAMANAMURThy and MATTI VUORINEN

A nonexistence result for the $n$-Laplacian

TILAK BHATTACHARYA

Bourgain algebras on the unit disk

Joseph A. Cima, Karel M. Stroethoff and Keith Yale

Lacunary statistical convergence

JOHN ALBERT FRIDY and CIHAN ORHAN

On the shape of fundamental domains in $\operatorname{GL}(n, \mathbf{R}) / \mathrm{O}(n)$

DOUGLAS MARTIN GRENIER

Fixed points of surface diffeomorphisms

BOJU JIANG and JIANHAN GUO

The moduli of rational Weierstrass fibrations over $\mathbf{P}^{1}$ : singularities

PABLO LEJARRAGA

On discrete isometry groups of negative curvature

GAVEN MARTIN

Adjoint linear systems on a surface of general type in positive characteristic

TOHRU NAKASHIMA

A homotopy transfer for finite group actions

WILLIAM J. RALPH

Maps between Seifert fibered spaces of infinite $\pi_{1}$

YONGWU RONG

Some numeric results on root systems

J. Y. SHI

Singular homology and cohomology with local coefficients and duality for manifolds

EDWIN SPANIER 